\title{
El fenómeno Paracas: estado de la cuestión y un modelo de explicación social
}

Artículos originales: ARQUEOLOGÍA

Recibido: $17 / 09 / 2020$

Aprobado: 04/11/2020

Henry Tantaleán

Publicado: 05/02/2021

\author{
Universidad Nacional Mayor de San Marcos \\ htantaleany@unmsm.edu.pe
}

\begin{abstract}
RESUMEN
En este artículo se presenta una síntesis de las principales investigaciones y evidencias arqueológicas relacionadas con el fenómeno Paracas de la costa sur peruana, el cual se desarrolló durante gran parte del primer milenio antes de nuestra era (800-200 a.C.). Se presentan y discuten las principales investigaciones, la cronología, el territorio que abarcó y los principales asentamientos asociados con la cultura material vinculada con este fenómeno social. Finalmente, se presenta un modelo de explicación social para comprender las dinámicas sociales que permitieron la aparición de lo que es conocido en la literatura arqueológica como la «Cultura Paracas».
\end{abstract}

Palabras clave: costa sur del Perú; Horizonte Temprano; Paracas; asentamientos; estilos cerámicos.

\section{The Paracas phenomenon: state of the art and a model of social explanation}

\begin{abstract}
This article presents a synthesis of the main investigations and archaeological evidence related to the Paracas phenomenon of the southern Peruvian coast, which developed during much of the first millennium before our era (800-200 BC). The main investigations, the chronology, the territory covered and the main settlements associated with the material culture linked to this social phenomenon are presented and discussed. Finally, a model of social explanation is presented to understand the social dynamics that allowed the appearance of what is known in archaeological literature as the «Paracas Culture».
\end{abstract}

KeYwords: Southern Peruvian coast; Early Horizon; Paracas; settlements; ceramic styles. 


\section{Introducción}

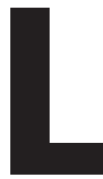

a sociedad Paracas ocupó un importante espacio geográfico de la costa sur peruana durante el I milenio antes de Cristo (800200 a. C.). Más allá de comprenderla como una "cultura arqueológica» con una misma forma de economía, política e ideología e integradas bajo una misma estructura política, en este artículo se evidencia y se propone que el fenómeno Paracas se trataría de una serie de comunidades con diferentes formas de organización económica y política pero que se vincularon económicamente y compartieron una misma ideología religiosa. Los mecanismos sociales por los cuales todas estas comunidades y entidades políticas se vincularon fueron el intercambio, el ritual y la religión, lo cual permitió que compartieran una serie de artefactos, prácticas sociales, rituales e iconografía religiosa. Como se verá, en cada uno de los valles y concentración de asentamientos Paracas, se puede reconocer que cada una de estas entidades o grupo de comunidades tuvieron sus propias manifestaciones arquitectónicas y artesanales y fueron autónomas económica y políticamente (Figura 1). Los famosos sitios arqueológicos asociados al Cerro Colorado en la península de Paracas parecen haber sido más que un lugar central para la sociedad Paracas, un espacio social de integración en el cual el culto a los ancestros destacó como sustento ideológico y religioso que permitió conectar comunidades y elites de diferentes espacios de la costa sur del Perú.

En este artículo se realiza una síntesis y análisis de la información arqueológica existente sobre la sociedad Paracas. Específicamente nos enfocaremos en lo que, en su momento, Julio C. Tello definió como «Paracas Cavernas». Aunque mencionaremos cuestiones relacionadas con lo denominado como «Paracas Necrópolis» o Topará, en este artículo no desarrollaremos una descripción y explicación de este fenómeno social diferente y posterior (Tello 2005[1959]: 201; Lanning 1960; Wallace 1986; Silverman 1991; Proulx 2008: 564). Asimismo, en este texto se plantea un modelo de explicación sobre la sociedad Paracas apoyándonos en la literatura existente y en nuestras propias investigaciones arqueológicas.

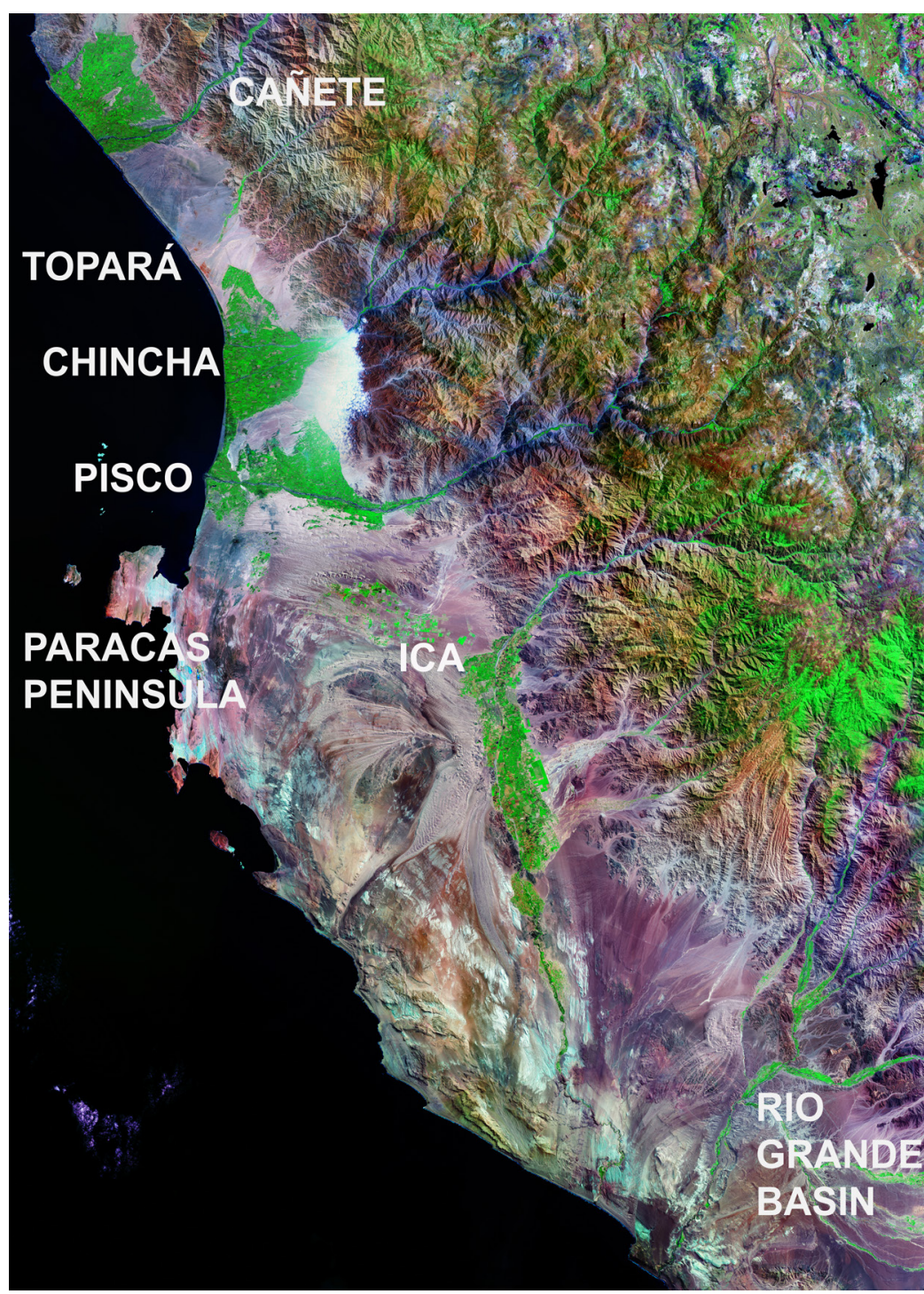

Figura 1: La costa sur del Perú. Vista Landsat, cortesía de Jalh Dulanto.

\section{Las investigaciones sobre el fenómeno Paracas}

Previamente a su descubrimiento científico, en sus exploraciones a comienzos del siglo XX de los valles de Ica y Chincha, el investigador alemán Max Uhle descubrió materiales y arquitectura que le resultaban más antiguos que lo que había visto previamente y que, por tanto, definió como "Proto-Nasca». De hecho, Uhle realizó excavaciones en 1900 en Huaca Alvarado un edificio del valle de Chincha (Uhle 1924: 81) y en 1901 en sitios de la hacienda Ocucaje del valle de Ica (Uhle 1914: 5), yacimientos que luego serán asignados a la cultura Paracas. 
Sin embargo, desde el punto de vista científico y arqueológico debemos el descubrimiento y definición de la "cultura Paracas" al investigador peruano Julio C. Tello. En 1925, acompañado por Samuel Lothrop y Rachel Warren y guiado por un huaquero local llamado Juan Quintana, conocedor de donde se venían extrayendo cerámica y textiles conocidos en el mercado internacional, Tello hizo el descubrimiento en las laderas del Cerro Colorado de una importante concentración de tumbas prehispánicas (Paul 1991: 5; Daggett 1991: 35). Sus excavaciones posteriores tuvieron como objetivo salvar los materiales arqueológicos, principalmente fardos con textiles y cerámicas polícromos del saqueo al que estaban expuestos. Entre 1925 y 1930, Tello se dedicó a las excavaciones de enterramientos junto con sus asistentes y colegas, principalmente Toribio Mejía Xesspe. En 1925, Tello descubrió las Cavernas en Cerro Colorado y Cabeza Larga (también conocido como Arena Blanca). En Cerro Colorado excavó 8 cavernas funerarias y 75 entierros individuales en fosas y en Cabeza Larga descubrió 66 enterramientos humanos (Tello y Mejía Xesspe 1979). En 1927, Tello descubre las «Necrópolis» de Wari Kayan de las cuales se retiraron 429 fardos funerarios. Gracias a sus investigaciones, Tello definió la existencia de dos «culturas»: Cavernas y Necrópolis (Tello y Mejía Xesspe 2005[1959]: 200-207). Dicha diferenciación se basaba en la forma de enterramiento y sus materiales asociados y los tipos de asentamientos que encontró vinculados a cada "cultura». De hecho, se trataban de dos zonas muy cercanas y que tenían una historia vinculada. Cavernas se refería principalmente a las tumbas subterráneas excavadas en los sedimentos endurecidos del mismo Cerro Colorado, incluidas su cima y laderas. Mientras que Necrópolis se refería a espacios arquitectónicos en los que se acomodó una gran cantidad de fardos y restos arqueológicos y que se localizaba en las laderas del Cerro Colorado y en el sector conocido como Cabeza Larga/Arena Blanca. Tello, además, visitó otros sitios relacionados con esta sociedad en la Bahía de la Independencia, los valles de Pisco, Ica y la cuenca del río Grande.

En 1931, Jorge C. Muelle y Eugenio Yacovleff prosiguieron con las excavaciones en el área de las Cavernas. Ellos descubrieron los mismos patrones funerarios ya definidos por Tello previamente. Muelle también investigó en los sitios de Karwas y Chucchio.

En 1925, Alfred Kroeber (1944: 137) realizó excavaciones en el valle de Chincha, concretamente en el sitio de Huaca Santa Rosa, la construcción más monumental del valle de Chincha.

Como parte de los trabajos de la Expedición de la Universidad de Columbia, William Duncan Strong (1957) realizó excavaciones entre 1951 y 1953 en Ocucaje, Teojate y Cahuachi. Guiado por Pablo Soldi, excavó en Teojate (que Strong denominó Juan Pablo I), un sitio en el valle alto de Ica encontrando 4 entierros de individuos colocados en posición sedente y relacionados con cerámica de la fase Paracas Temprano.

Entre 1955 y 1959, Frederic Engel realizó intensas prospecciones de la costa sur localizando una importante serie de sitios Paracas, incluidos muchos de ellos en la misma península. Engel, incluso, realizó excavaciones en los sitios Cabeza Larga y Karwas (Engel 1966, 1991). Asimismo, Engel reportó en el valle medio de Pisco, en el área cercana al sitio Inca de Tambo Colorado, sitios domésticos con materiales cerámicos Paracas (Engel 1957). En las décadas de 1950 y 1960, Alejandro Pezzia también realizó excavaciones en el valle de Ica (1968).

Entre 1955 y 1961, los integrantes de la denominada «Escuela de Berkeley» liderada por John Rowe, realizaron intensas prospecciones y excavaciones en el valle de Ica (Menzel et al. 1964: iii). Especialmente, se enfocaron en dicho valle con el objetivo de generar una «Secuencia Maestra» para los Andes prehispánicos. Dorothy Menzel y Lawrence Dawson se encargaron de darle contenido material a las fases de dicha secuencia, incluso realizando pequeñas excavaciones en sitios del valle (Menzel et al. 1964: iii). Por su parte, Dwight Wallace realizó importantes prospecciones en los valles de Chincha, Pisco, Cañete e Ica, encontrando muchos sitios Paracas. Asimismo, junto a Edward Lanning, Wallace visitó y excavó sitios Paracas en el valle de Chincha. Wallace también excavó en el sitio de Cerrillos en el valle de Ica en 1958 (Wallace 1962), que suministró evidencias estratigráfícas para afinar la secuencia Paracas (Menzel et al. 1964: 1). Las excavaciones en Cerrillos lideradas por Wallace continuaron entre 1999 y 2003 (Splitstoser 2009; Splitstoser et al. 2009). 
En la década de 1980, Sarah Massey, Anita Cook y Lisa DeLeonardis investigaron en el valle de Ica. Massey se enfocó entre 1980 y 1981 en el reconocimiento de los sitios del valle alto de Ica y, posteriormente, en los sitios del sector de Callango del mismo valle (Massey 1991: 315-316). Asimismo, realizó excavaciones en 1982 en el sitio de Ánimas Altas (Massey (1982, 1991). En dicho sitio, Massey descubrió un friso con decoraciones complejas plasmado en la pared de una pequeña plataforma. Entre 1988 y 1990, Cook se concentró en la prospección de sitios del valle bajo de Ica (Cook 1999: 61). DeLeonardis se enfocó en los sitios de la sección de Callango del valle de Ica (DeLeonardis 1997, 2005).

En la misma década, Luis Lumbreras y su equipo realizaron prospecciones y excavaciones en el valle de Chincha. Entre 1985 y 1987 se enfocaron en la excavación del sitio El Mono o Chococota (Peters 1987/1988: 30). De esos trabajos, resultó la tesis de maestría de Elizabeth Isla que aporta importantes datos sobre la cerámica Paracas del valle de Chincha.

Por su parte, Ann Peters prospectó el valle de Pisco en 1985 con el objetivo de completar el reconocimiento realizado por Wallace. Asimismo, en 1987 Peters realizó excavaciones en el sitio de Chongos, un sitio perteneciente a Paracas y Topará que Tello y Mejía Xesspe ya habían visitado previamente (Peters 1987/1988, 2013). Helaine Silverman (1997) también trabajó en la parte baja del mismo valle de Pisco, en el sitio Alto de Molino. Silverman también trabajó a finales de la década de 1980 en el valle de Ingenio, encontrando sitios especialmente en su sección alta relacionados con Paracas y que definió como parte de su estilo Tajo (Silverman 1994).

En la década de 1990 aparecieron algunos artículos de José Canziani describiendo sus análisis de la arquitectura Paracas del valle de Chincha tomando como base investigaciones del equipo de Lumbreras del cual formó parte.

Por su parte, en la década de 1990 el arqueólogo peruano Rubén García realizó investigaciones en diversos sitios de la Península y de la Bahía de la Independencia (García y Pinilla 1995, García 2010). Sus trabajos en el sitio Puerto Nuevo son importantes para comprender los momentos más tempranos de Paracas en esta zona (García 2009).

Desde 1997, los trabajos del equipo alemánperuano dirigido por Markus Reindel y Johny Isla han aportado importantes datos para el valle de Palpa relacionados con la ocupación Paracas. Su proyecto multidisciplinar ha reportado ocupaciones Paracas con asentamientos, geoglifos y petroglifos. Asimismo, ha generado una importante cantidad de fechados radiocarbónicos que permitieron establecer una cronología absoluta más detallada para Paracas (Unkel et al. 2012).

Dentro de un proyecto de investigación financiado por el gobierno alemán que también prospectó el sector superior del valle del Rio Grande a partir del 2006, Peter Kaulicke y asociados (2009), investigaron una serie de tumbas en el sitio de Coyungo (BRiG 3117). Allí encontraron tumbas Paracas Temprano con materiales significativos entre los que sobresalen fragmentos de textiles con decoración pintada similares a los encontrados en Karwas.

Desde el año 2007 y hasta la actualidad, Aïcha Bachir Bacha y Daniel Llanos han dirigido un programa arqueológico que se ha concentrado en el sitio Ánimas Altas/Bajas (Bachir-Bacha y Llanos 2013, Bachir Bacha 2017). Su investigación ha profundizado en la naturaleza y dinámica social de uno de los sitios más extensos de la sociedad Paracas. Asimismo, excavaron en otro de los sitios más importantes del área de Ocucaje: Cerro Max Uhle o Córdova (Llanos 2017).

En la década de 2010 varios proyectos arqueológicos que han estudiado la sociedad Paracas han surgido en la costa sur. En el valle de Ica entre 2012 y 2017, el «One River Project» liderado por Charles French, David Beresford-Jones y Kevin Lane y conformado por arqueólogos británicos y peruanos, realizó intensas prospecciones en el valle de Ica, reportando numerosos sitios, algunos de ellos con ocupaciones Paracas. Dentro de ese proyecto, también se reportaron sitios Paracas en la parte serrana de Ica (2000 $\mathrm{msnm})$ con rasgos similares a los reportados en Palpa. Por su parte, Masato Sakai y su equipo durante su prospección arqueológica de las pampas de Nazca han descubierto geoglifos asociados a cerámica Paracas. Las investigaciones lideradas por Pedro Castro-Martínez (2016) en el valle de Aja también han contribuido al entendimiento de Paracas en el extremo sur, un área que había sido previamente investigada por Hendrik van Gijseghem (2006) y Juan Carlos de la Torre. Jalh Dulanto y Aldo Accinelli (2013) y su equipo de la Pontificia Universidad 
Católica del Perú revisitó sitios descubiertos por Engel y excavó en yacimientos del área de la península de Paracas como Disco Verde y Puerto Nuevo. Finalmente, desde el 2011, Charles Stanish y Henry Tantaleán han desarrollado trabajos extensivos e intensivos en el valle de Chincha. Han excavado en sitios Paracas como Cerro del Gentil, Chococota, Huaca Soto, La Cumbe y Pozuelo (Tantaleán et al. 2013; Stanish et al. 2014, Tantaleán y Stanish 2017).

\section{Cronología del fenómeno Paracas}

Como señalamos, en la década de 1960 se planteó una cronología relativa para Paracas conocida como la "Secuencia de Ocucaje» la cual fue dividida en 9 fases cerámicas (Menzel et al. 1964). Dicha secuencia cerámica ha servido como marco para organizar los estilos y fases cerámicas, aunque también hay que reconocer que existen algunos desfases e inadecuaciones en otros valles de la Costa Sur. Asimismo, por problemas en el mínimo tamańo de la muestra y apoyándose en la evidencia radiocarbónica disponible, varios investigadores (Paul 1991: 31; Wallace 1991: 103; Silverman 1991: 378) han descartado la existencia de las dos primeras fases.

Los trabajos de Engel permitieron obtener fechados radiocarbónicos que posibilitaron obtener una primera cronología general del desarrollo de los Paracas en la costa sur, en especial, de los sitios arqueológicos de la Bahía y la península de Paracas y de la Bahía de la Independencia (Engel 1991, García y Pinilla 1995, León 2007). Adicionalmente, otros fechados obtenidos por Dulanto (2013; Dulanto y Accinelli 2013) han sustentado la cronología absoluta de sitios como Disco Verde y Puerto Nuevo. Finalmente, recientes fechados fueron obtenidos del colágeno de dientes de individuos enterrados en Cerro Colorado, Wari Kayan y Karwas (PezoLanfranco et al. 2015).

Otros proyectos arqueológicos enfocados en sitios del valle de Ica, en especial de la ocupación Paracas (Splitstoser et al. 2009, DeLeonardis 2005, Cadwallader et al. 2015), obtuvieron más fechados radiocarbónicos, siempre correlacionándolos con la secuencia desarrollada por Menzel, Dawson y Rowe.

Por su parte, Tantaleán y Stanish (Tantaleán et al. 2013) para el valle de Chincha, Kaulicke y asociados
(2009) para el valle del rio Grande y Pedro Castro y su equipo (2016) para el valle de Aja, han ofrecido fechados radiocarbónicos que permiten tener una secuencia mucho más detallada de eventos ocurridos fuera del valle de Ica.

No obstante, los trabajos del Proyecto NazcaPalpa han encontrado que la mayoría de las fases propuestas por Menzel y colegas encajan dentro de las fases encontradas en sus sitios arqueológicos y que también han podido ser fechadas (Unkel et al. 2012). De este modo, recientemente, una importante cantidad de fechados radiocarbónicos (41 fechados) obtenidos de las investigaciones en sitios del valle de Palpa ha permitido establecer una cronología absoluta para Paracas (Unkel et al. 2012: 2301). Esta se divide en Paracas Temprano (840-500 a. C.), Medio (500-380 a. C.) y Tardío (380-260 a. C.). De acuerdo a esta cronología, Paracas Temprano se correlaciona con las Fases 3 y 4 de la Secuencia Cerámica de Ocucaje, Paracas Medio se correlacionaría con la Fases 5 a 7 y, finalmente, Paracas Tardío con las Fases 8 y 9 .

\section{El territorio del fenómeno Paracas}

El territorio sobre el cual se extendió el fenómeno Paracas se trata de una región costera desértica de una extensión longitudinal de unos 250 kilómetros sobre la cual discurren valles que traen aguas desde la cordillera andina. De norte a sur, los valles que se vinculan con la sociedad Paracas son: Cañete, Topará, Chincha, Pisco, Ica y los valles que pertenecen a la Cuenca del rio Grande (Palpa, Ingenio, Nazca, Aja). Asimismo, la humedad del mar que se condensa en ciertas zonas genera lomas estacionales. En general, la franja costera se encuentra entre el litoral del Océano Pacífico y los inicios de la cordillera andina. Como se verá, grupos sociales vinculados directamente a Paracas también han sido localizados en las partes altas de los valles en la zona de cordillera hasta una altura de $3200 \mathrm{msnm}$ (Reindel e Isla 2018).

De acuerdo a propuestas precedentes (Massey 1986: 308-311; Silverman 1997: 456; Isla y Reindel 2007; Bachir Bacha 2017: 220, 2018: 5) y nuestras recientes investigaciones, el territorio de los Paracas no sería homogéneo y, más bien, se podría dividir en 
diferentes áreas. En nuestro caso, planteamos que el territorio Paracas se podría dividir en tres áreas: norte, centro y sur. Dichas áreas tendrían como límites, principalmente, a las zonas desérticas y montañosas existentes entre los valles. De este modo, el epicentro de los Paracas del norte sería el valle de Chincha, el cual se vincularía con los valles de Cañete, Topará y Pisco, el de los Paracas del centro estaría focalizado en el valle de Ica y, finalmente el de los Paracas del Sur, cuya zona principal estaría en el valle de Palpa y que se relacionaría directamente con los valles de los ríos Grande, Ingenio y de la Cuenca de Nazca. Aunque dichas regiones tienen importantes vinculaciones y áreas de contacto como pueden ser los sitios de la península de Paracas (Pezo-Lanfranco et al. 2015), los de la Bahía de la Independencia (García y Pinilla 1995) e, incluso el mismo valle de Ica, estas tres grandes áreas mantienen ciertas diferencias especialmente con relación a sus respectivas formas de asentamiento, diseños arquitectónicos y técnicas constructivas, formas de enterramiento y cultura material. Como zona de contacto principal, los sitios de Cerro Colorado de la península de Paracas conforman un lugar muy especial y en el que convergerían varias de las comunidades de los valles arriba mencionados, principalmente de los valles de Ica y Chincha. Por tanto, por ser el lugar epónimo de la cultura Paracas y el más conocido, empezaremos con la descripción de este complejo arqueológico para luego ir de norte a sur describiendo cada uno de los valles y zonas significativas del territorio Paracas (Figura 2).

\section{Los sitios de la península de Paracas}

Los principales sitios de la zona de Paracas son Cerro Colorado, Wari Kayán y Cabeza Larga/Arena Blanca. Como señalamos, las Cavernas de Cerro Colorado fueron las primeras estructuras funerarias descubiertas científicamente por Tello. Se trata de una extensa zona de enterramientos de aproximadamente 8 hectáreas localizada sobre terrazas ubicadas en la parte superior del cerro (Figura 3). (Pezo-Lanfranco et al. 2015: 24). En esa área, Tello y sus colegas excavaron 8 cavernas funerarias y unas 75 tumbas hechas en fosa (Tello y Mejía 1979). Las cavernas fueron excavadas, en algunos casos hasta la profundidad de 7 metros, en el substrato sedimentario endurecido (Figura 4). En el interior de la cámara de forma ovoidal, a la

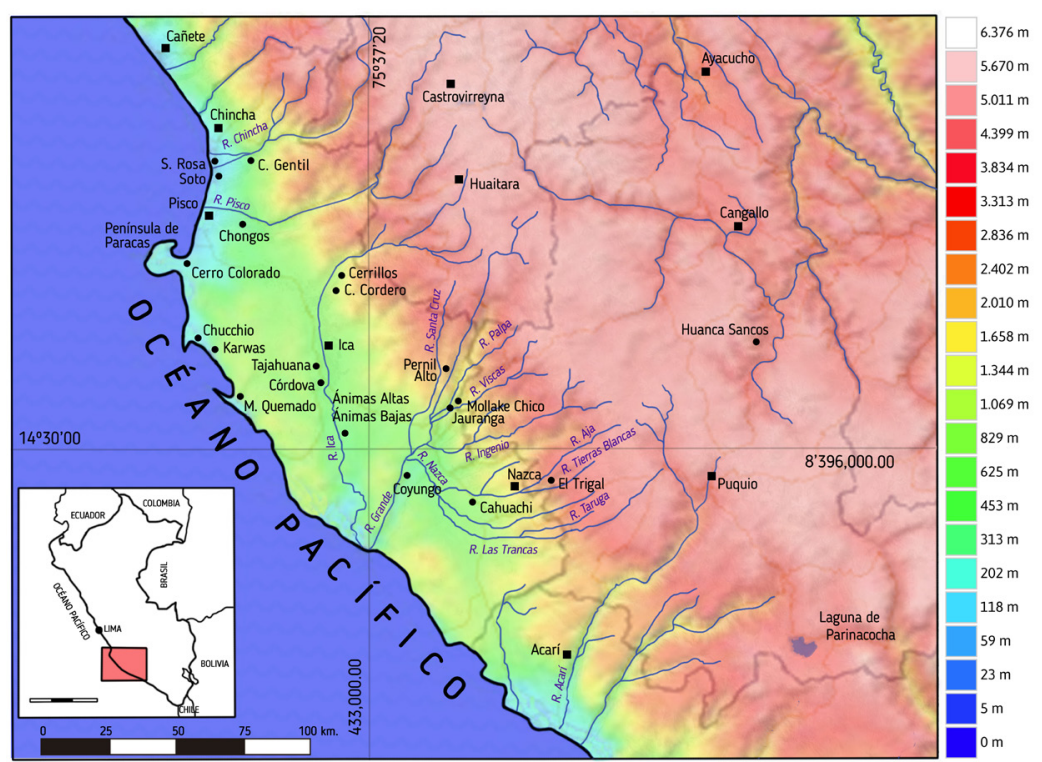

Figura 2. Mapa de la costa sur con los principales sitios mencionados en este texto.

que se accedía tras atravesar una antecámara y conducto tubular, se hallaron concentraciones de fardos funerarios compuestos por capas de textiles que envolvían cadáveres (hasta 53 individuos en el caso de la Caverna V). Dentro de los fardos se encontraban individuos en posición sedente, con las piernas y los brazos flexionados contra el torso. Los cuerpos estaban acompañados de vasijas cerámicas, textiles, cestas de junco y otros artefactos (Tello 2009). Además, existían enterramientos en fosas de hasta 3 metros de profundidad que también incluían la inhumación de fardos funerarios (Tello y Mejía Xesspe 1979). Se calcula que entre todas esas tumbas se recuperó más de medio millar de enterramientos (Pezo-Lanfranco 2015: 25).

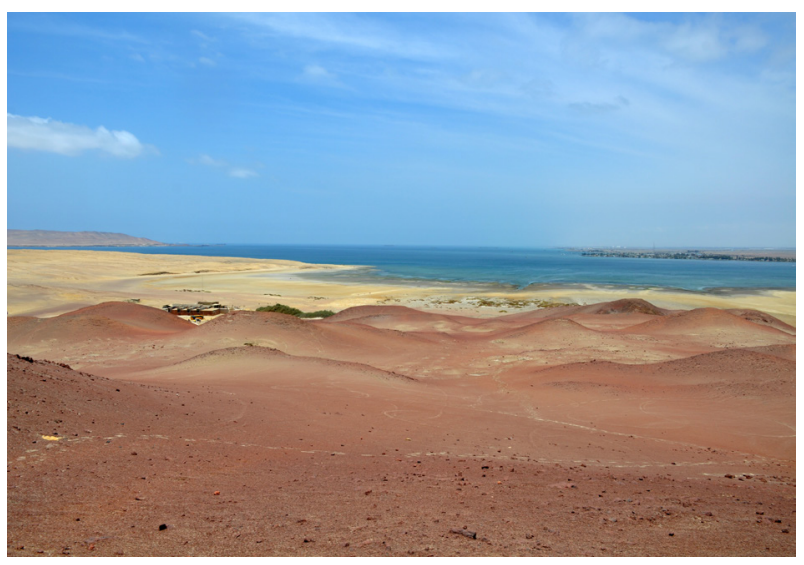

Figura 3. Vista de Cerro Colorado. 


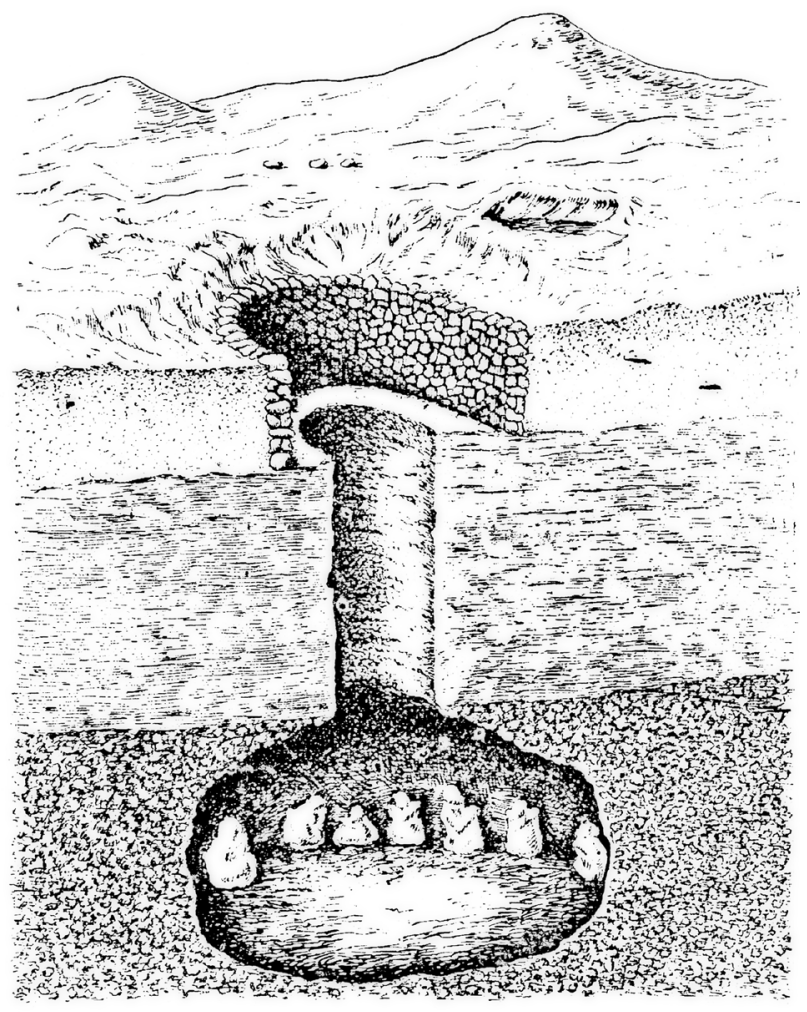

Figura 4. Caverna Paracas en Cerro Colorado. Modificado de Tello 1929.

En la ladera norte del Cerro Colorado, se extienden las «Necrópolis de Wari Kayan", así denominadas por Tello (Tello y Mejía 2005[1959]; Tello y Mejía 1979). Se trata de la reutilización para fines funerarios de una serie de estructuras rectangulares semisubterráneas con muros de piedra. Es muy probable que dichas estructuras arquitectónicas habrían sido utilizadas como viviendas y depósitos por poblaciones relacionadas con los grupos que enterraron a sus muertos en las Cavernas (Silverman 1991: 354; Pezo-Lanfranco 2015: 25). Según el mismo Tello (1979: 76), este sector doméstico abarcaría unos $40,000 \mathrm{~m}^{2}$. Aunque los entierros realizados en Wari Kayan incluyen algunos especímenes y enterramientos de época Paracas Tardío, la mayoría pertenece a la fase Necrópolis o Topará (Dwyer 1979: 109). Hasta 1930, Tello y su equipo recuperaron en este sector 429 fardos funerarios.

Finalmente, la otra gran concentración de estructuras arquitectónicas y enterramientos se encuentra en el sector de Arena Blanca o Cabeza Larga. Este sector se extiende en la parte baja de una ladera y la llanura cercana a la playa. Según Tello (1979: 249)

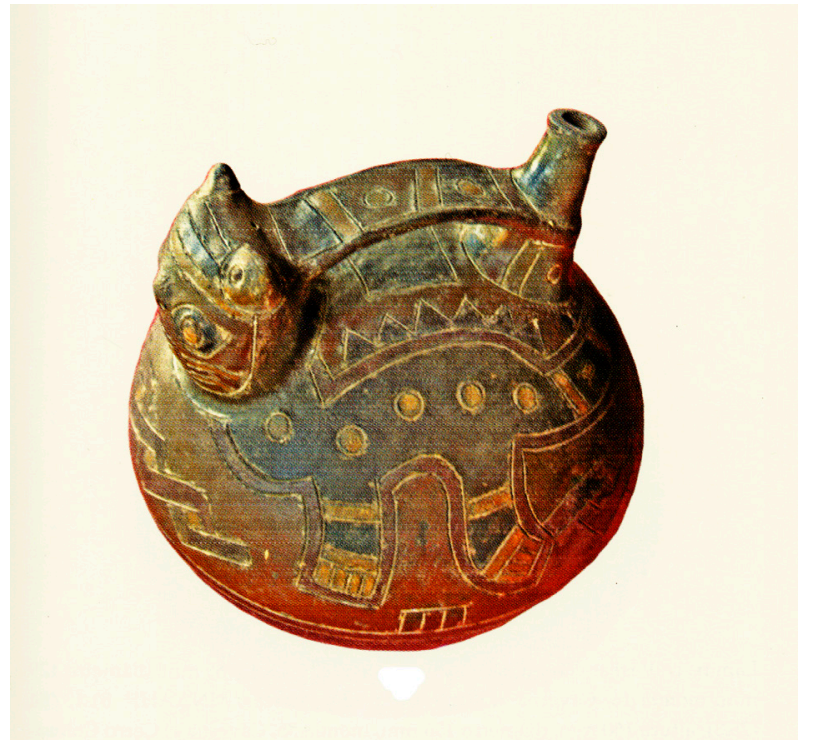

Figura 5. Vasija cerámica Paracas excavada en la Caverna VI de Cerro Colorado. Tomado de Tello 1959.

este sitio se extiende por 54 hectáreas. Se trata de una concentración de estructuras habitacionales realizadas con muros hechos de piedra sobre la cual existe una gran concentración de restos de consumo de alimentos y actividad humana. Asimismo, se han recuperado enterramientos humanos también de la tradición funeraria Paracas Cavernas pero, sobre todo, posteriores (Tello 2009, Dwyer 1979: 108; Engel 1991).

El principal indicador arqueológico de la sociedad Paracas, como fue definido en Cerro Colorado, son las vasijas cerámicas decoradas con incisiones, decoración negativa y pintura resinosa polícroma aplicada poscocción sobre la superficie de las vasijas (Silverman 1991: 356). Los diseños incorporan seres antropomorfos, zoomorfos y diseños geométricos (Figura 5). Esta tradición de vasijas cerámicas se extiende para todos los sitios Paracas que se describirán en este texto. Asimismo, existe una tradición de textiles de algodón y lana decorados con motivos antropomorfos y zoomorfos, especialmente serpientes entrelazadas y diseños geométricos (Dwyer 1979; Silverman 1991: 360). Otros materiales hallados en las tumbas Paracas de la península son artefactos de metal (oro y cobre), mates pirograbados, cestas, estólicas, bastones de madera, lanzas de madera, flechas, puntas de obsidiana, porras de piedra, hondas, redes de pesca, artefactos con plumas, entre otros. Finalmente, se debe señalar la presencia de modifi- 
caciones craneanas entre los individuos enterrados en Cerro Colorado. Algunos de los cráneos presentaron trepanaciones craneanas.

\section{El valle de Cañete}

El reconocimiento de Wallace en el valle medio de Cañete realizado en 1958 (1963) ubicó 4 sitios con cerámica Paracas. Entre ellos, resalta el sitio Patos de cuya superficie se recuperaron fragmentos cerámicos Paracas que Helaine Silverman (1991: 380) ha identificado como similares a los de la fase Ocucaje 8. El sitio Patos se trata de una aldea con estructuras arquitectónicas hechas con bases de piedra (Wallace 1986: 42).

\section{El valle de Topará}

La prospección de Wolfgang Wurster y su equipo (1984) sólo identificó 2 sitios con ocupación Paracas. Se trata de pequeños asentamientos domésticos que tuvieron fragmentos de cerámica Paracas en superficie. A pesar de su cercanía con el valle de Chincha no se registraron edificaciones monumentales Paracas.

\section{El valle de Chincha}

El valle de Chincha posee una de las principales concentraciones de edificios monumentales vinculados a la sociedad Paracas. Los sitios del valle bajo son principalmente monumentales y se encuentran en el fondo del valle, salvo La Cumbe que se encuentra en el borde del tablazo que domina la parte norte del valle. Entre esas edificaciones podemos mencionar a La Cumbe, Huaca Limay, Huaca Alvarado, Huaca Santa Rosa, Huaca Soto, los Complejos Soto y San Pablo (Canziani 1992; Tantaleán 2016) (Figura 6). Los edificios tienen plantas rectangulares y están orientados en un eje oeste-este y poseen como rasgo principal una serie de plazas amuralladas o patios hundidos. Para su construcción se utilizaron adobes de barro hechos a mano y algunas veces cantos rodados. Huaca Santa Rosa es la edificación monumental más importante del valle y de toda la costa sur de época Paracas con un largo de 400 metros, un ancho promedio de 150 metros y una altura de unos 15 metros. En el caso de los sitios del valle medio estos son más pequeños y se encuentran ubicados al borde de las

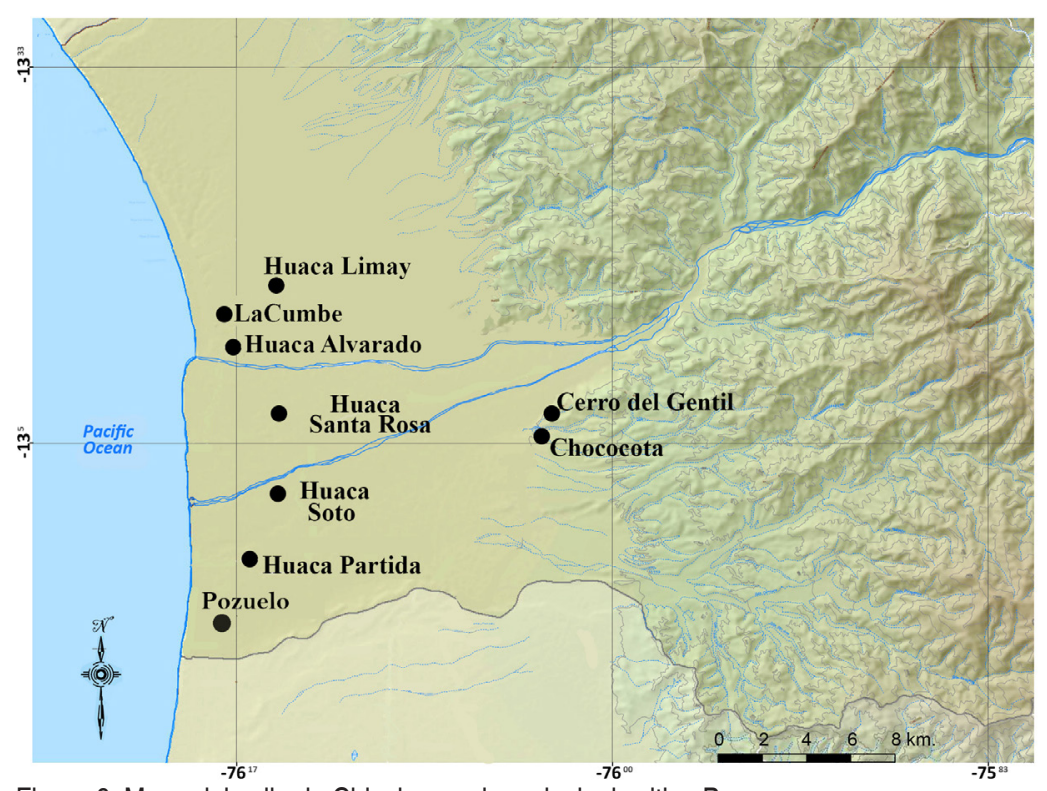

Figura 6. Mapa del valle de Chincha con los principals sitios Paracas.

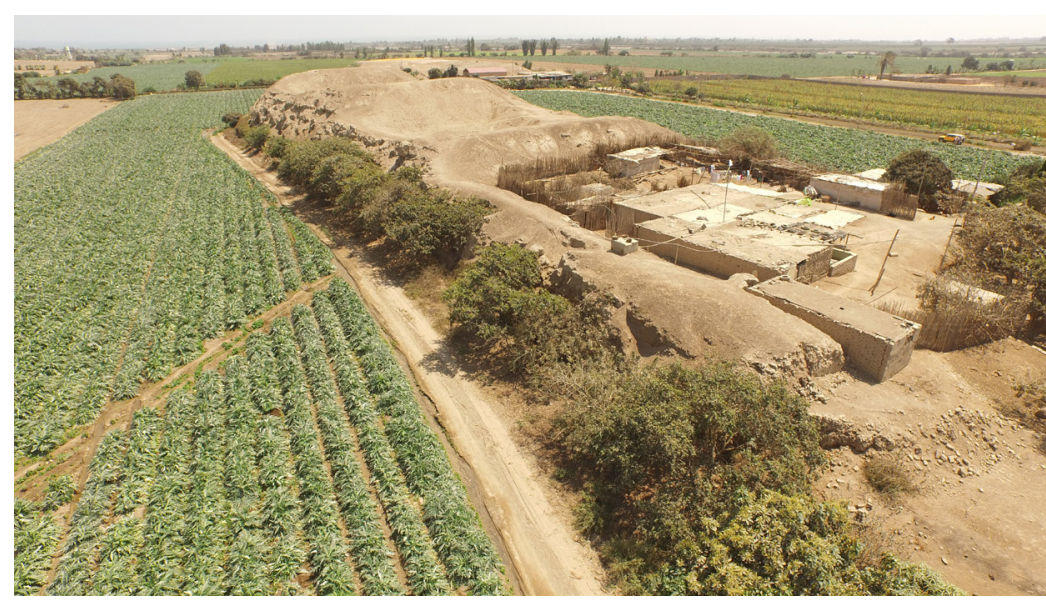

Figura 7. Huaca Soto.

pampas que se elevan sobre el fondo del valle. Entre estos sitios tenemos a Cerro del Gentil y el Complejo El Mono (Tantaleán et al. 2013). Adicionalmente se han localizado pequeños asentamientos domésticos como Portachuelo y áreas domésticas alrededor de Huaca Soto enterradas varios metros debajo de la superficie actual (Figura 7). Sin embargo, a diferencia de otros valles como el de Ica o Palpa, no se han descubierto concentraciones de estructuras domésticas. Quizás, como en el caso del Complejo Soto, estas ocupaciones se encuentren sepultadas por metros de sedimento. Uno de los pocos asentamientos Paracas conocidos de carácter doméstico es el de Pozuelo. 
Los edificios Paracas más tempranos del valle de Chincha surgieron en el VIII siglo antes de Cristo como es el caso de Huaca Soto (Nigra 2017). Asimismo, el sitio de Pozuelo tiene una ocupación desde el siglo IX y vinculado al estilo conocido como Pozuelo que se ha vinculado con la fase Ocucaje 3 y con el estilo Puerto Nuevo (García 2009; Tantaleán et al. 2019). De este modo, todos estos sitios se encontrarían dentro de la fase Paracas Temprano. Sin embargo, entre los siglos VI y III una serie de edificios públicos fueron levantados. Entre ellos resaltan Cerro del Gentil, El Mono y La Cumbe. Pozuelo también siguió siendo ocupado. Por los reconocimientos y excavaciones se puede relacionar a otros edificios como Huaca Santa Rosa, Huaca Alvarado y el complejo San Pablo con estos edificios. Así, la principal concentración de edificios en el valle de Chincha se daría durante las fases Paracas Medio y Paracas Tardío.

La cerámica de los sitios Paracas del valle de Chincha es relativamente similar, especialmente la registrada en los sitios de Cerro del Gentil, La Cumbe y Huaca Santa Rosa. Se trata de vasijas principalmente decoradas en negativo y con incisiones y pintura poscocción. En el caso de Cerro del Gentil gracias la inhumación de individuos en fardos y en posición sedente, con las piernas y los brazos flexionados contra el torso, depósito de ofrendas y concentración de restos de consumo de alimentos durante eventos especiales poseemos una importante cantidad de cultura material (textiles, mates pirograbados, etc.). Estos restos arqueológicos guardan muchas similitudes con los hallados en las cavernas de la península de Paracas (Tantaleán y Stanish 2017). Asimismo, en la pampa al este de Cerro del Gentil y el Complejo El Mono se han identificado geoglifos lineales que estarían asociados con los sitios Paracas de la zona (Stanish et al. 2014).

\section{El valle de Pisco}

El sitio Paracas más importante del valle de Pisco es Chongos. A pesar que la mayor parte del sitio pertenece a la ocupación Topará, también tuvo una ocupación Paracas previa (Silverman 1991; Peters 2013). Otro sitio del valle bajo con evidencias Paracas es Alto del Molino (Silverman 1997: 453). Adicionalmente, Engel (1957) señaló la existencia de sitios Paracas Tardío en el valle medio, cercanos a Tambo Colorado. En este caso, se trata de asentamientos domésticos y tumbas ubicadas en las laderas de los cerros. Finalmente, durante recientes trabajos de arqueología de contrato realizados en el valle medio se hallaron enterramientos Paracas Medio (Balbuena 2013).

\section{El valle de Ica}

El valle de Ica se ha dividido en valle alto, medio y bajo. Al norte de la ciudad de Ica se encuentra el inicio del valle alto. Hacia el sur de esa misma ciudad se extiende el valle medio, en el que resalta el sector de Ocucaje. Más al sur de Ocucaje se extiende el valle bajo que tiene como principal protagonista al sector de Callango. Otros sectores de la parte baja del valle, que incluyen a Ullujaya y Samaca, se extienden hacia el sur hasta llegar a la desembocadura del rio.

\section{Valle Alto de Ica}

\section{Cerrillos}

En la margen este del valle alto de Ica se localiza el sitio de Cerrillos, uno de los primeros sitios Paracas en ser excavados científicamente (Wallace 1962). Se trata de un extenso sitio ubicado en una ladera de cerro que domina este sector del valle y que concentra terrazas habitacionales con muros de piedra. Sin embargo, el principal hallazgo fue el descubrimiento de un edificio público («Templo») con múltiples remodelaciones o fases arquitectónicas que fue construido con adobes de barro hechos a mano y que incluían recintos y escalinatas (Figura 8). Las diferentes fases arquitectónicas se dieron lo largo de gran parte del I milenio a.C. Dichas remodelaciones también fueron fechadas mediante radiocarbono (Splitstoser et al. 2009: 210). Se evidenció que las primeras fases arquitectónicas de Cerrillos estuvieron relacionadas a la fase Ocucaje 3 y las últimas a Ocucaje 9 (Splitstoser et al. 2009: 210). Asimismo, durante la ocupación Paracas Temprano se evidenció la ocurrencia de artefactos foráneos, principalmente cerámicos. Algunos de ellos presentaban rasgos "chavinoides» (Splitstoser et al 2009: 214). Adicionalmente, durante su fase Paracas Temprano se han hallado textiles muy semejantes en técnica y decoración a los de Karwas y Coyungo (Splitstoser et al. 2009: 227). 


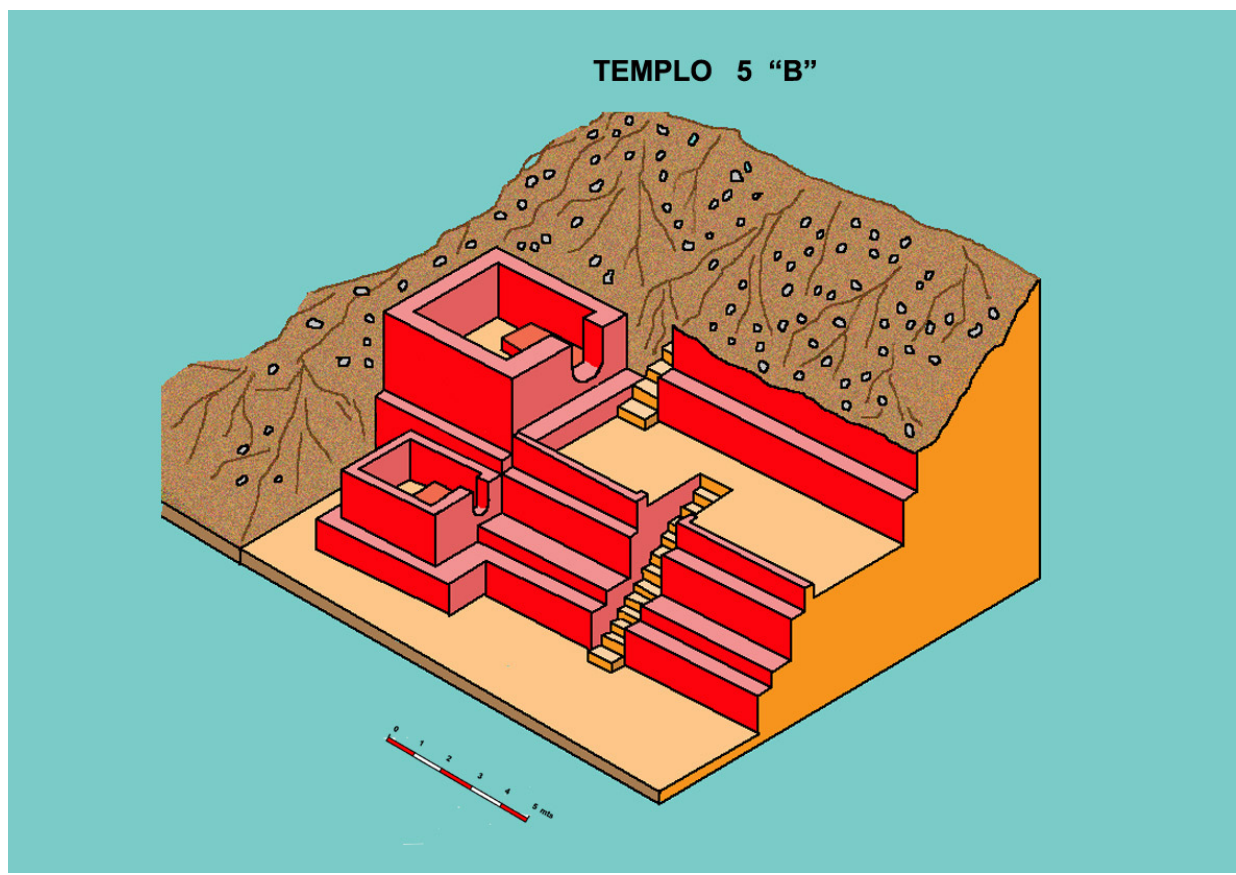

Figura 8. Reconstrucción arquitectónica del edificio principal de Cerrillos. Cortesía de Dante Casareto.

\section{Teojate}

El otro sitio importante de este sector del valle en su margen oeste es el cementerio de Teojate ubicado en la base del cerro del mismo nombre. Este sitio fue denominado por William D. Strong con el nombre de Juan Pablo. Procede de dicho cementerio una de las mejores colecciones de vasijas Paracas y que fueron detalladamente analizadas por Alan Sawyer (1966) (Figura 9). Se trataría de un cementerio para las diferentes comunidades de esta parte del valle (DeLeonardis 2005: 37). El cementerio fue utilizado desde Paracas Temprano hasta Paracas Tardío (Menzel et al. 1964: 55, 75, 102; DeLeonardis 2005: 37).

Adicionalmente, Kevin Lane y sus colegas (2017) han investigado sitios Paracas en el valle alto de Ica, localizados a 2,000 msnm. Los asentamientos comparten rasgos arquitectónicos con los encontrados por Reindel e Isla en la sierra de Palpa.

\section{Valle Medio de Ica}

Anita Cook (1999: 69) identificó 51 sitios Paracas en su prospección del valle de Ica que incluyó los sectores de Ocucaje y Callango. Los sitios van desde Paracas Temprano (Ocucaje 3) hasta Paracas Tardío (Ocucaje 9). Por lo general, los asentamientos Paracas no ocupan áreas inmediatas al rio o el fondo del valle y están construidos con adobes y quincha.

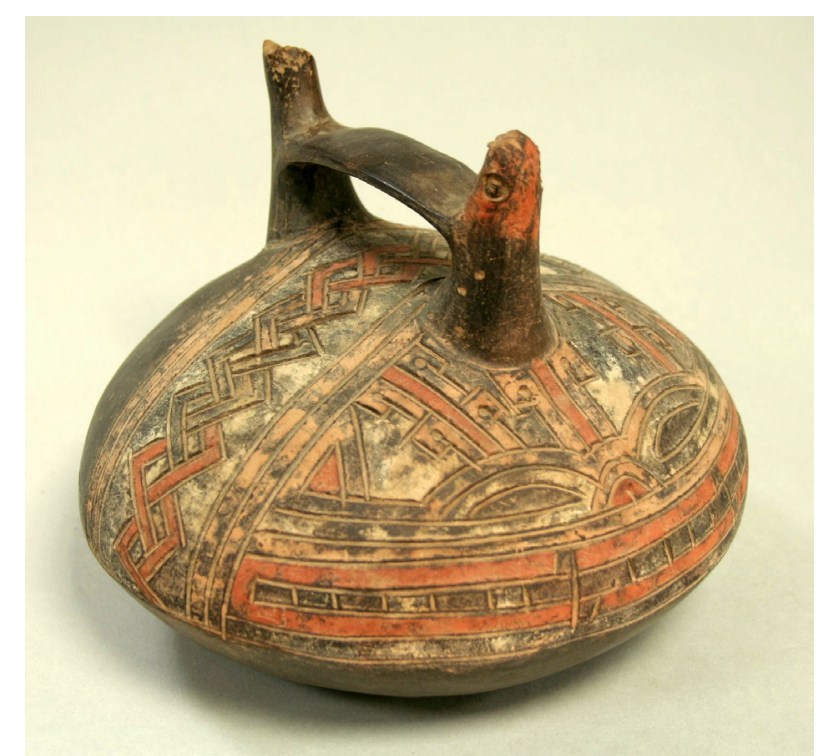

Figura 9. Vasija cerámica excavada en Teojate. Metropolitan Museum of Art.

\section{Sector de Ocucaje}

De acuerdo a Cook (1999: 70), los sitios habitacionales Paracas se encuentran ubicados en la cima de cerros alejados del río. En el área específica de Ocucaje existe una serie de sitios arqueológicos con ocupación Paracas (Cerro Córdova o Max Uhle, La Peña, Pinilla). Cerro Córdova fue excavado en 1901 por Max Uhle y, posteriormente, por Daniel Llanos y 


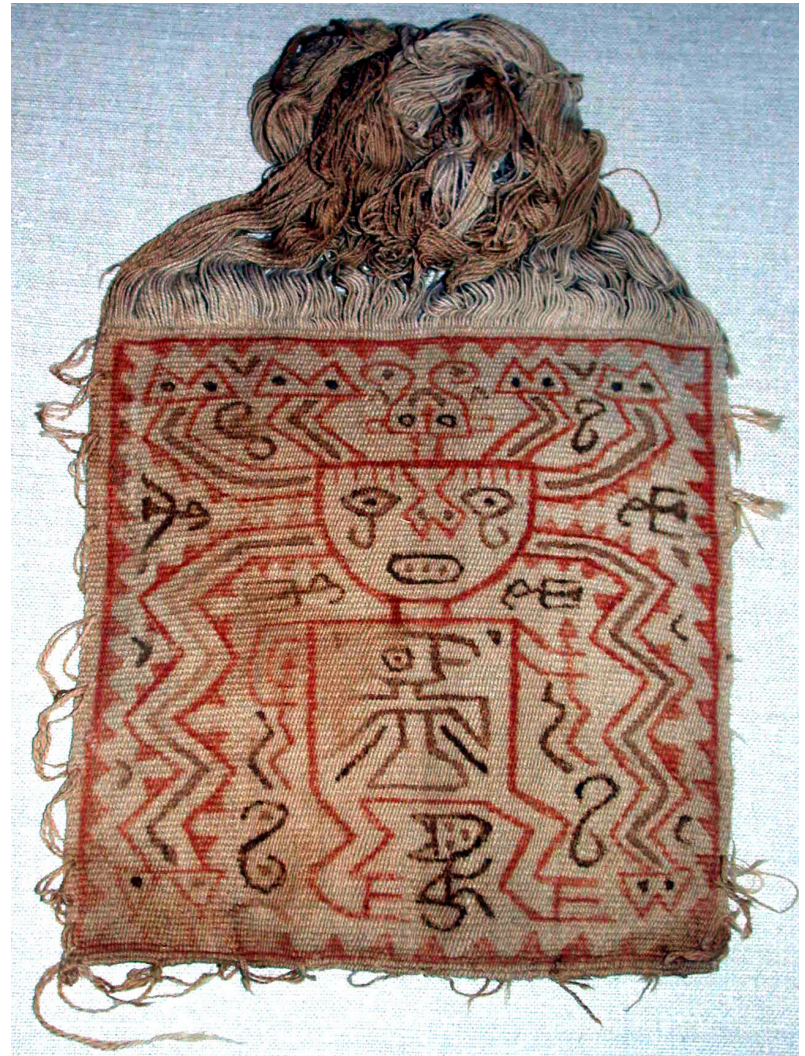

Figura 10. Máscara de tela de fardo funerario de Ocucaje. Metropolitan Museum of Art.

Aïcha Bachir Bacha. Sus ocupaciones se vinculan a la fase Ocucaje 9 de Paracas. Asimismo, hay ocupaciones de la fase 10 y Nazca 1. Cerro Córdova es el sitio más extenso de Ocucaje con arquitectura pública y

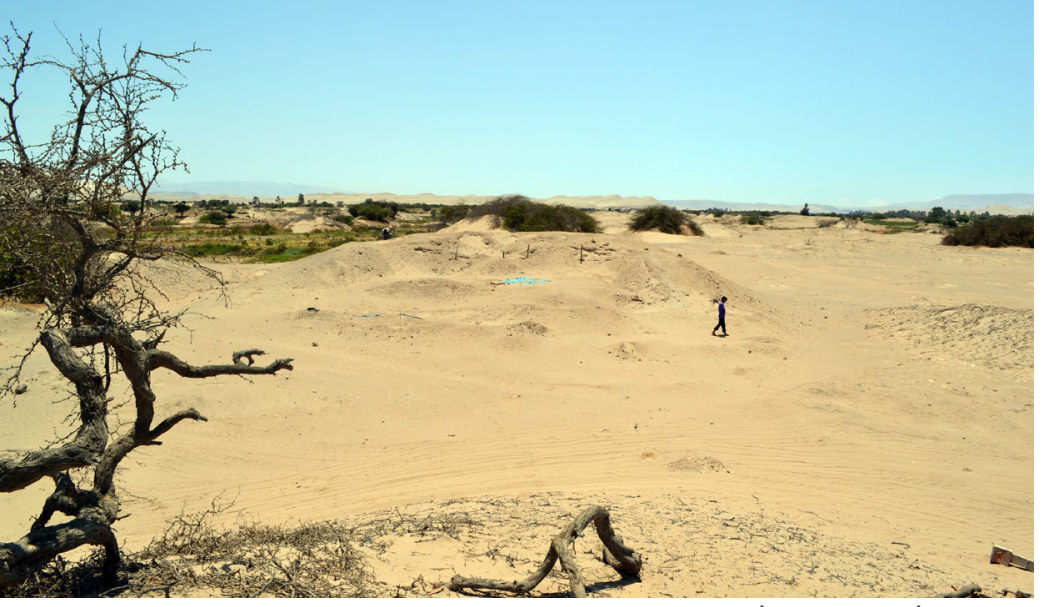

Figura 11. Vista del Edificio de los Frisos de Ánimas Altas/Ánimas Bajas. que, posiblemente, concentró el poder económico y político después del decaimiento de Ánimas Altas/ Ánimas Bajas. El sitio La Peña se convirtió en un centro público ceremonial también de la fase Paracas Tardío y se ubica en un cerro en cuyas laderas se construyeron terrazas habitacionales (Cook 1999: 74).

La excavación de una serie de cementerios en Ocucaje ha proporcionado cerámica decorada para todas las fases Paracas (Menzel et al. 1964). En especial, de estos cementerios se han recuperado máscaras de fardos funerarios de telas pintadas, las cuales solo han sido encontradas en estos sitios Paracas y pertenecen a la fase 9 y 10 de Ocucaje (Dawson 1979) (Figura 10). Adicionalmente, entre Ocucaje y Callango, Sawyer (1966: 74) reporta el hallazgo de vasijas cerámicas con diseños chavinoides en el sitio de Chiquerillo y que deben pertenecer a Paracas Temprano. Incluso, Lisa DeLeonardis (2005) señala que este sitio debió tener ocupación del periodo Inicial.

\section{Valle Bajo de Ica}

\section{Sector de Callango}

En el sector de Callango, los sitios fueron construidos en las zonas llanas alejadas del fondo del valle. DeLeonardis (2005) llevó a cabo una prospección y excavaciones reportando 26 sitios Paracas desde Ocucaje 3 hasta 10. De entre todos ellos, el Complejo Ánimas Altas/Ánimas Bajas es el sitio Paracas más extenso del valle, ocupando una extensión de 90 hectáreas y reuniendo un centenar de montículos de una altura máxima de 7 metros (Bachir Bacha 2017: 194). Algunos de esos montículos han demostrado ser edificaciones públicas en las cuales se realizaban rituales. Asimismo, existen estructuras residenciales y talleres. Las edificaciones fueron construidas con muros hechos con bloques de barro. Se ha establecido que sus ocupaciones humanas estuvieron vinculadas a las fases 8 a 10 de Ocucaje (Bachir Bacha 2013). De acuerdo a ello, se plantea que la ocupación Paracas se desarrolló entre los 400 y 100 a. C. En ese sitio, Sarah Massey (1991: 325) descubrió frisos en la fachada de un edificio público (Figura 11). Los trabajos de Bachir Bacha y Llanos (2017) han extendido la exposición de dicho friso y han encontrado otros más. Los frisos representan a seres antropomorfos, en especial al «Ser Oculado» (Figura 12). De hecho, se- 

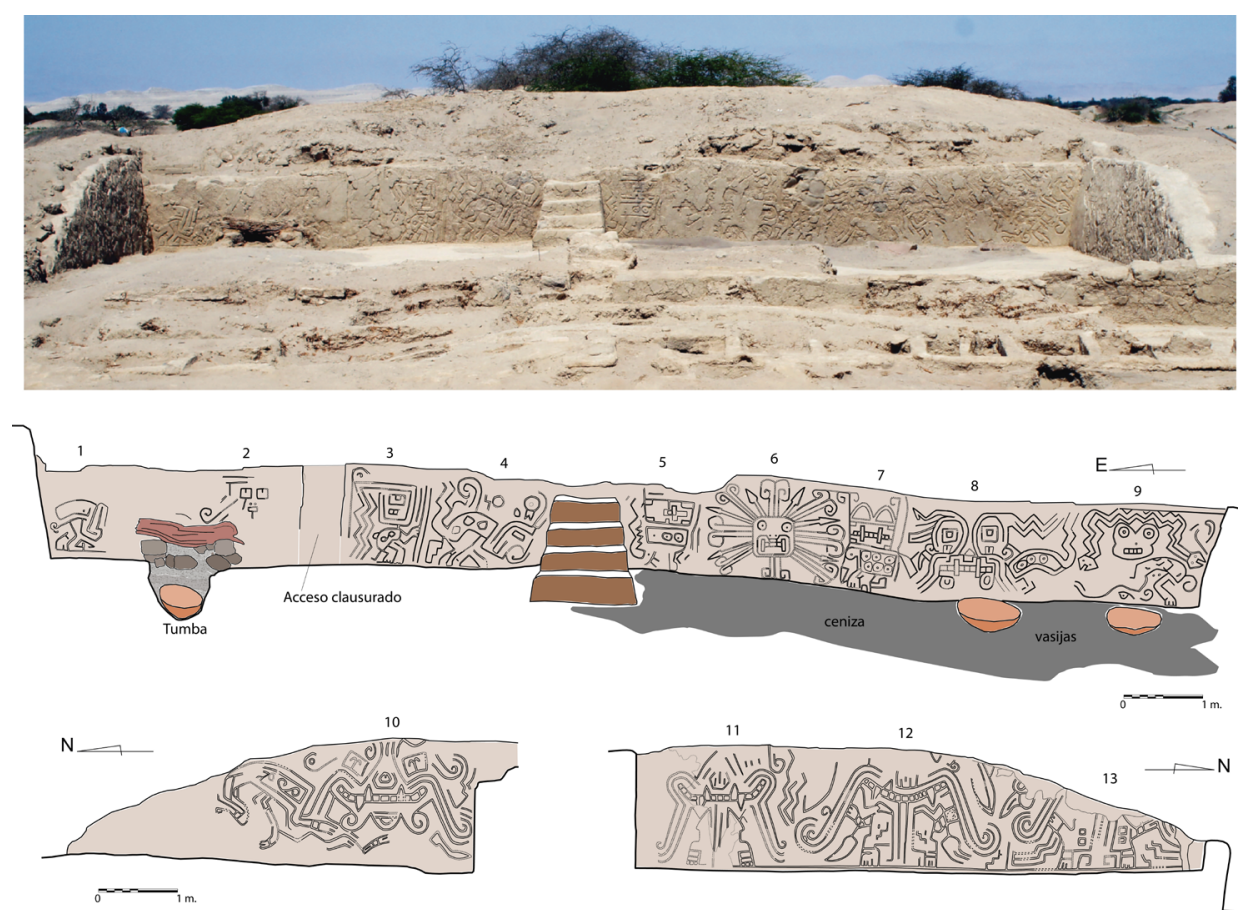

gún Llanos (2017: 186), Ánimas Altas/Ánimas Bajas se convirtió en el centro del culto de dicho personaje.

En el sector al sur de Callango denominado Ullujaya el equipo del «One River Project» localizó un sitio (Ullujaya G-8/9) asociado a fragmentos cerámicos de las fases Ocucaje 3 y 4 (Beresford-Jones et al. 2009: 241). Además, en Samaca se ubicó el sitio 1004 que está vinculado a las fases Ocucaje 9 y 10 (Cadwallader et al. 2018). Asociado a este sitio se ha reportado un conjunto de geoglifos lineales.

\section{Sitios de la Bahía de la Independencia}

De acuerdo a una serie de investigadores (García y Pinilla 1995, Cook 1999: 74, DeLeonardis 2005), dos de los principales sitios de la Bahía de la Independencia, Karwas y Chucchio, estaban vinculados con poblaciones del valle de Ica durante la época Paracas. Incluso, en áreas ubicadas entre la ruta del valle de Ica a la Bahía de la Independencia se ha registrado geoglifos de época Paracas (García 2013; Isla 2018: 117).

\section{Karwas}

El sitio de Karwas se encuentra al sur de la Bahía de la Independencia en una amplia zona de playa asociada a la Punta Carhuas, un peńasco rocoso que penetra en el mar. El sitio se localiza sobre una terraza natural adyacente al mar y se extiende, por al menos, 28 hectáreas (García 2010: 59). Aunque el sitio fue descubierto por Tello en 1927 y se hallaron enterramientos de Paracas muy similares a los de Cerro Colorado, el sitio se volvió famoso por el hallazgo en la década de 1960 de, al menos, 200 fragmentos de textiles de algodón pintados con motivos vinculados con la ico-

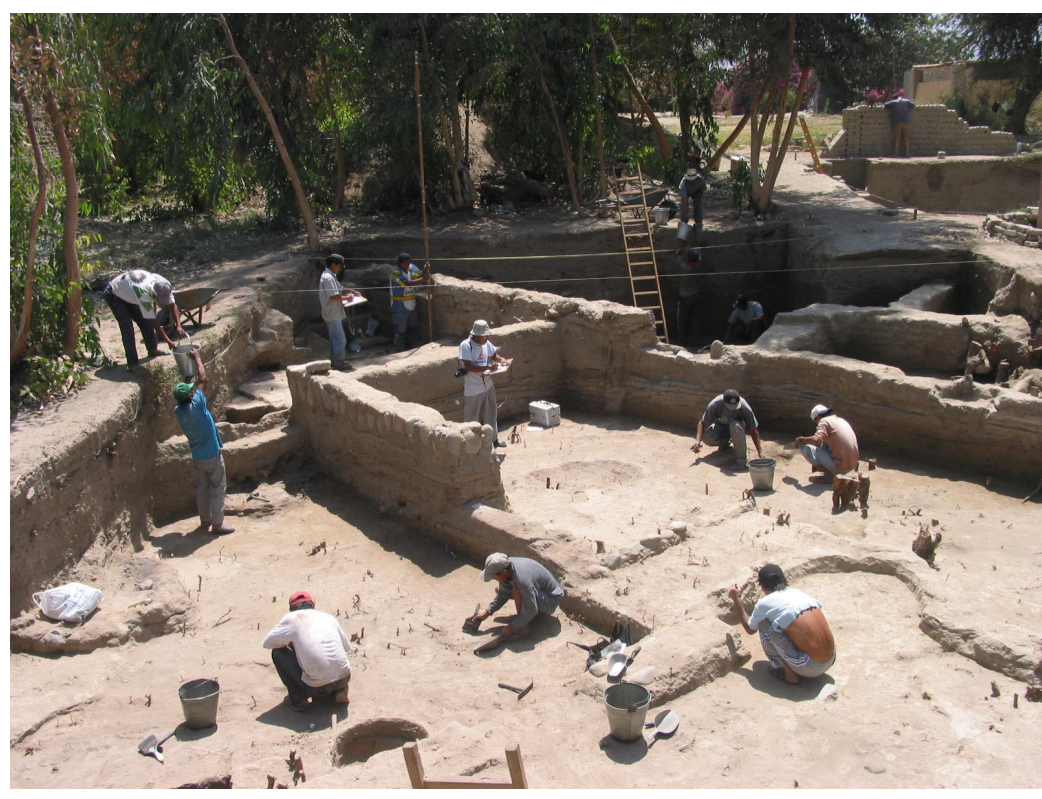

Figura 13. Vista de Jauranga. Cortesía de Markus Reindel y Johny Isla. 
nografía de Chavín de Húantar, principalmente inspirados en el "dios de los báculos» (García y Pinilla 1995). Por tanto, el sitio se vincula principalmente a la fase Ocucaje 3, aunque también hay cerámica Paracas Tardío (Wallace 1991; García y Pinilla 1995; García 2010: 59). El sitio se trata de una concentración de estructuras arquitectónicas, basurales y tumbas, muchas de ellas saqueadas (García 2010: 59).

\section{Chucchio}

El sitio de Chucchio se encuentra también cercano a la playa, a 10 kilómetros al norte de Karwas. Se trata de un asentamiento doméstico de unas 30 hectáreas y compuesto por unos 20 montículos. Para la construcción de los muros principales se utilizaron cantos rodados (García 2010: 57). La cerámica relacionada a las estructuras arquitectónicas pertenece a Paracas Tardío (García 2010: 58).

\section{El valle de Palpa}

El valle de Palpa contiene una de las principales concentraciones de asentamientos Paracas de toda la costa sur alcanzando un total de 150 sitios vinculados a dicha sociedad (Reindel e Isla 2013: 233). La gran mayoría, un total de 118 asentamientos, pertenecen a la fase Paracas Tardío (Reindel 2009: 448). Los sitios van desde la parte inferior del valle a $300 \mathrm{msnm}$ hasta los $3000 \mathrm{msnm}$ en la cordillera andina. Los sitios se ubican, sobre todo, en las zonas áridas ubicadas en los márgenes del valle y las laderas de los cerros, aunque unos pocos se han ubicado en el fondo del valle (Reindel 2009: 446). Las estructuras arquitectónicas están, principalmente, construidas con piedra y quincha y, en algunos casos, con adobes de barro. Los sitios Paracas van desde Ocucaje 3 hasta Ocucaje 10 (Isla y Reindel 2007: 82). La agricultura se dio sobre las zonas naturalmente irrigadas por el rio y no se han evidenciado sistemas hidráulicos (Reindel 2009: 448). El equipo de Reindel e Isla han realizado excavaciones en una serie de sitios Paracas. De entre ellos, destacan Pernil Alto, Mollake Chico, Jauranga y Pinchango Viejo.

\section{Pernil Alto}

Se trata de un asentamiento con estructuras arquitectónicas cuadrangulares hechas de barro construidas durante el período Inicial (Reindel 2009). En un momento posterior se inhumaron a cinco individuos relacionados con cerámica de la fase Ocucaje 3 y, por tanto, evidenciando la ocupación Paracas Temprano del sitio (Isla 2009: 125). Los individuos fueron enterrados decúbito dorsal y de forma extendida y acompañados con vasijas cerámicas. Además, de estos enterramientos también se han ubicado estructuras habitacionales cercanas que pertenecieron a esta misma fase. Resulta interesante que, a diferencia de otros sitios Paracas, en el caso de Pernil Alto los individuos estuvieron colocados decúbito dorsal y extendidos.

\section{Mollake Chico}

Este sitio se encuentra en un área ubicada en el margen del rio, en la parte inferior del valle de Palpa. En Mollake Chico se descubrió una tumba colectiva Paracas Temprano (Ocucaje 3). Dentro de la estructura funeraria rectangular de 2.5 de largo por $1.8 \mathrm{~m}$ de ancho se hallaron 17 individuos (12 adultos y 5 niños e infantes) (Isla y Reindel 2007:83). Los restos de los individuos inhumados mostraban signos de cremación, una práctica que no había sido reportada previamente para los enterramientos Paracas (Reindel 2009:445).

\section{Jauranga}

Se trata de un asentamiento residencial ubicado en tierras del fondo del valle medio de Palpa (Figura 13). Sus ocupaciones van desde la fase Ocucaje 5 hasta 10. Vale decir, incluyen ocupaciones Paracas Medio y Paracas Tardío (Reindel 2009). En este sitio se han hallados 49 entierros Paracas, la mayoría pertenecientes a la fase Paracas Tardío (Ocucaje 8). Como en el caso de Mollake Chico, los individuos mayoritariamente fueron colocados de forma extendida y decúbito dorsal y existen evidencias de un ritual de cremación de los restos humanos (Reindel 2009: 447, 449). Este sitio podría haber sido uno de los principales lugares de producción cerámica del valle (Reindel e Isla 2013: 256).

\section{Pinchango Viejo}

Se trata de un asentamiento Paracas Tardío ubicado en el valle medio de Palpa. Sobre las laderas y base de un cerro se han construido una serie de terrazas habitacionales. Varias murallas restringen el acceso al asentamiento. Se hallaron enterramientos humanos en posición sedente y cerámica de la fase Ocucaje 8 (Reindel 2009: 450). 
Finalmente, vale la pena mencionar una serie de sitios Paracas Tardío encontrados arriba de los 3000 msnm (Reindel e Isla 2018). Se trata de asentamientos en cumbres como el de Cutamalla con una arquitectura que en vista de planta posee «forma de flor» (Reindel e Isla 2017). Se trataría de sitios residenciales vinculados a la producción de camélidos, fibras y textiles.

Además de los asentamientos mencionados, el equipo de Reindel e Isla ha identificado petroglifos en las localidades de Chichictara con motivos similares a los de la iconografía Paracas. Asimismo, en los valles de Palpa se encuentran geoglifos Paracas Tardío como los de Llipata (Isla y Reindel 2007) (Figura 14).

\section{El valle de Ingenio}

Las prospecciones realizadas por Silverman (1994) ubicaron una serie de asentamientos Paracas. De entre esos sitios, ubicados a lo largo del valle de Ingenio, destaca la concentración de sitios en la parte media del valle. El sitio Tajo es el que le dio nombre a un estilo Paracas definido por Silverman. Sin embargo, investigaciones recientes han demostrado que se trata de un tipo de cerámica que corresponde a cerámica doméstica ubicada en otros tantos sitios Paracas de la costa sur.

\section{El valle del Río Grande}

El sector superior del valle del rio Grande ubicado entre la confluencia de dicho rio con el rio Nazca y la localidad de Las Brujas fue prospectado en el 2006 por un equipo alemán dirigido por Burkhard Vogt y en el cual participó Peter Kaulicke (Kaulicke et al. 2009). Allí se registraron una serie de asentamientos Paracas. Entre ellos, destaca el sitio de Coyungo.

\section{Coyungo}

En este sitio se investigaron una serie de tumbasmausoleos Paracas Temprano (Kaulicke et al. 2009). Se excavaron 4 tumbas colectivas construidas con barro y materiales botánicos, las cuales en conjunto reunieron los restos de un mínimo de 24 individuos. Entre la gran cantidad de materiales locales y exóticos (cerámica, mates modificados, puntas de obsidiana, etc.) resalta el hallazgo de textiles pintados con

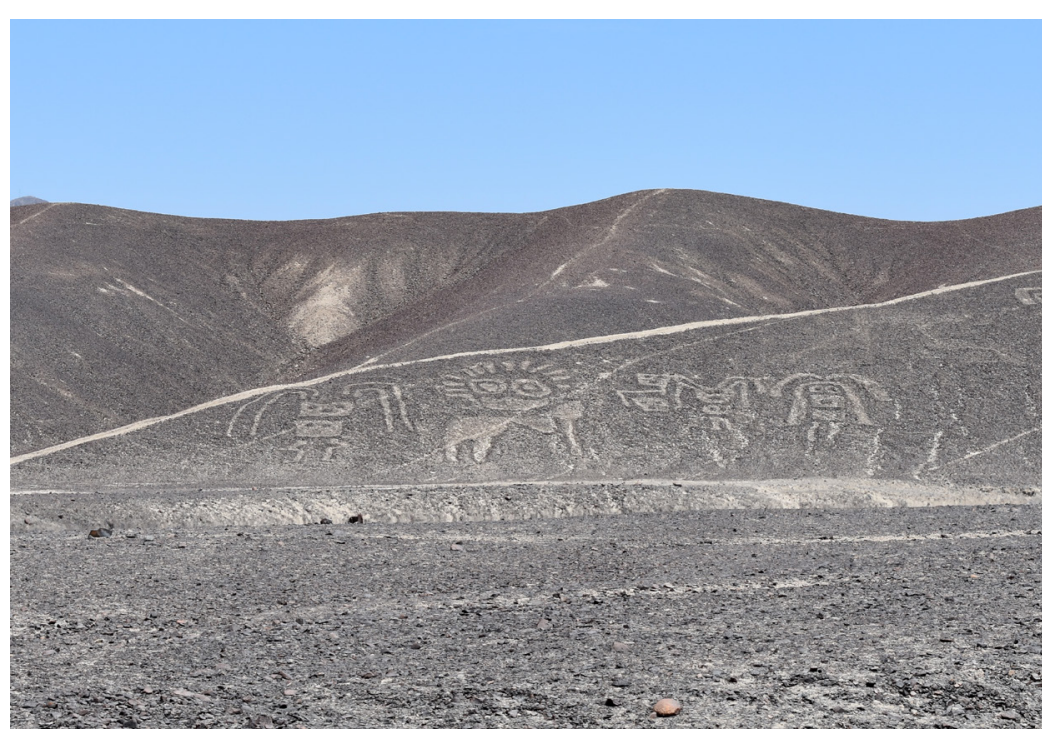

Figura 14: Geoglifos de Llipata.

diseños chavinoides muy similares a los hallados en Karwas.

\section{El valle de Nasca}

Para el valle de Nazca solamente tenemos referencias de ocupaciones Paracas en el importante sitio de Cahuachi.

\section{Cahuachi}

William D. Strong (1957) señaló tempranamente la presencia de cerámica Paracas en este sitio. Posteriormente, diferentes investigadores han señalado que, más bien, se trataba de cerámica de la fase Ocucaje 10 (Menzel et al. 1964: 3; Paul 1991: 372; Van Gijseghem 2006: 427). Pese a ello, las investigaciones de Giuseppe Orefici en este sitio han recuperado cerámica de las fases Ocucaje 9 y 10 (Orefici 2012, Tomo I: 74, 76). Adicionalmente, en las pampas de Nazca, ubicadas entre los valles de Ingenio y Nazca, el equipo liderado por Masato Sakai ha registrado geoglifos asociados a fragmentos de cerámica Paracas Tardío (Sakai et al. 2014; Sakai et al. 2019).

\section{Valle de Aja}

El área arqueológica de La Puntilla concentra una serie de asentamientos sobre las cimas y laderas de unos cerros muy cercanos a la confluencia de los ríos 
Aja y Tierras Blancas. El sitio de La Puntilla que se extiende por unas 3 hectáreas tiene arquitectura de muros de piedras y su cerámica corresponde a las fases Ocucaje 8 y 9 (Van Gijseghem 2006).

Cerro de El Trigal es otro los asentamientos del área arqueológica de La Puntilla y que ha venido siendo investigado en los últimos ańos. Las investigaciones han demostrado que el sitio fue ocupado entre los 700 y 100 a. C., vale decir, desde la fase Paracas Temprano hasta la fase Paracas Tardío (Castro et al. 2016).

\section{Presencia de cerámica Paracas en otros sitios}

En el sitio de Ancón, localizado a unos 35 kilómetros al norte de la ciudad de Lima en la bahía del mismo nombre, Ernesto Tabío (1965: 74) identificó fragmentos de cerámica Paracas entre sus materiales excavados en el sector denominado Las Colinas. Al norte del valle de Mala, en un sitio muy cercano a la playa La Ensenada se hallaron enterramientos con cerámica y textiles Paracas Medio (Balbuena 2013). Para el extremo sur de la costa peruana, en el valle de Acarí se ha reportado el hallazgo de fragmentos cerámicos Paracas Tardío en sitios del valle como, por ejemplo, Hacha (Valdez 2012).

Desde la década de los 1970 en adelante, diferentes investigadores reportaron la ocurrencia de fragmentos de cerámica Paracas en varios sitios de la sierra central y sierra centro sur. De este modo, tenemos reportes de cerámica Paracas Temprano en Campanayuq Rumi, Ayacucho y Atalla, Huancavelica ((Matsumoto 2019; Young 2017) y Paracas Tardío en Huancayo, Junín y Huanta y Vilcashuamán en Ayacucho (Browman 1970: 64; Lumbreras 1974: 78; Mendoza 2017).

\section{Un modelo de explicación social del fenómeno Paracas}

Los Paracas se trata de un conjunto de comunidades con diferentes formas económicas y políticas que compartieron una ideología religiosa. A lo largo del tiempo se aprecia que algunas comunidades desarrollaron formas económicas pero, sobre todo, políticas que les permitieron controlar sus sectores de valle o todo un valle.
Por una parte, la independencia se evidencia hacia dentro de las comunidades y se basaba en el desarrollo de estrategias económicas, políticas y religiosas que les permitieron reproducir al grupo social y mantener sus propias identidades locales. Por otra parte, la interdependencia es una serie de relaciones externas a los grupos, comunidades o entidades políticas. En primer lugar, esta interdependencia se dio solamente entre elites y se focalizó en lugares centrales, en los cuales se reunía a una importante cantidad de personas y en los cuales se realizaban practicas colectivas relacionadas con cultos. En segundo lugar, está relación se amplió a otros miembros de cada comunidad y, a la vez, las vinculó con otras elites establecidas en sitios principales de la región de la costa sur. Asimismo, además de ser lugares de intercambio económico y de información y generación de relaciones sociales, estos lugares centrales eran importantes espacios sagrados relacionados con el culto (huacas) y, especialmente, a la veneración de los ancestros, como bien puede haber sido el caso de las cavernas de Cerro Colorado. La atracción de las elites y comuneros hacia esos sitios se basaba en la capacidad de las elites locales de generar condiciones materiales, espacios rituales y significados para cada lugar especial, huaca o conjunto de huacas. Asimismo, se fundamentaba en la capacidad de los líderes o elites para movilizar a sus propios comuneros y peregrinos para la realización de trabajos colectivos. En el caso de la economía de cada valle, esta capacidad se basaba en las estrategias económicas. Para ello, se desarrollaron actividades marisqueras, pesqueras, ganaderas y agrícolas. Así, por ejemplo, en el caso de los sitios de Cerro Colorado se ha evidenciado que sus habitantes consumían tanto alimentos de origen marino como agrícolas. En el caso de las comunidades del valle de Chincha se desarrollaron importantes proyectos hidráulicos en el valle bajo con el consecuente incremento en la producción agrícola pero también incorporaron alimentos marinos, especialmente mariscos. Además, hay presencia de camélidos. En el caso del valle de Ica se aprecia el aprovechamiento de las avenidas de los ríos para la producción agrícola y también tuvieron acceso a recursos marinos y ganaderos. De acuerdo a la información disponible, actividades similares fueron desarrolladas en los valles de Palpa. Esta serie de estrategias económicas, políticas y religiosas se dieron a diferentes niveles e intensidades. Así podríamos 
plantear las siguientes fases del desarrollo de esta independencia e interdependencia como sigue:

\section{Fase I}

Relacionada a los primeros momentos de Paracas, en este caso lo conocido como Paracas Temprano (840-500 d. C.). Se trata de comunidades autónomas que surgieron cerca al litoral o en los valles. Algunos de ellos sobre sustratos sociales del periodo Inicial. Así, tenemos sitios como Disco Verde y Puerto Nuevo en el litoral de la Bahía de Paracas (García y Pinilla 1995; Dulanto 2013; Dulanto y Accinelli 2013), Karwas en la Bahía de la Independencia (Wallace 1991), Pozuelo en el valle de Chincha (Lanning 1960; Tantaleán et al. 2019), sitios en el sector de Ocucaje (Menzel et al. 1964) y Cerrillos (Splitstoser et al. 2009; Splitstoser 2014) en el valle de Ica, Pernil Alto en el valle de Palpa (Reindel e Isla 2006) y Coyungo en el valle del Río Grande (García y Pinilla 1995; Tantaleán et al. 2019; Reindel 2009: 446, Kaulicke et al. 2009). Estos sitios tuvieron cerámica vinculada a la fase denominada Ocucaje 3 la cual también incorpora cerámica con rasgos «chavinoides» (Menzel et al. 1964).

En esta fase, si bien dichas comunidades fueron autónomas económica y políticamente, a nivel económico también compartían y se beneficiaban del culto de Chavín e ingresaron en su «esfera de interacción» (sensu Burger 1992:211). Sin embargo, también mantuvieron su independencia local y formaron sus propias versiones del culto Chavín. Aun los valles no se encuentran densamente habitados. De este modo, la cerámica Paracas surge sobre la base de una tradición local con una influencia y contacto externo, principalmente de la Costa Norte (Cupisnique) y de Chavín (Menzel et al. 1964, García y Pinilla 1995). Por ello, su cultural material posee sus propios matices.

\section{Fase II}

Esta fase está relacionada a un momento de independencia de la influencia Chavín y se daría a partir de su declive en el 500 d. C. Así, se extiende en lo conocido como Paracas Medio (500-380 a. C.). En este caso, las comunidades autónomas generan lo conocido como Paracas con sus propias particularidades. Es, en este momento, cuando las elites locales de cada valle comienzan a generar alianzas y los seguidores en cada valle comienzan a concentrarse en sus sitios principales que son lugares de intercambio económico y de culto. Sitios como las huacas del valle de Chincha surgen como edificios monumentales entre los que tenemos a Cerro del Gentil, Huaca Soto y, muy posiblemente, Huaca Santa Rosa, el Complejo San Pablo y Huaca Alvarado (Tantaléan et al. 2013; Nigra 2017). En el valle medio de Ica, en el sector de Ocucaje se desarrollan asentamientos que comienzan a concentrar el poder económico y político mientras que en el valle alto de Ica el sitio de Cerrillos sigue concentrando el liderazgo de esta zona (Wallace 1962; Menzel et al. 1964). Por su parte, los sitios de Palpa presencian un incremento de la construcción y sus enterramientos incorporan cerámica fina (Reindel 2009).

\section{Fase III}

Este momento está vinculado con Paracas Tardío (380-260 a. C.) y en la secuencia de Ocucaje, con las fases 8 y 9 . Este es el momento de mayor interdependencia entre las elites y sus seguidores en la costa sur. Los sitios de Cavernas de Cerro Colorado se convierten en lugares de culto a los ancestros y de intercambio de bienes de prestigio con artefactos de valles cercanos y de regiones alejadas de la costa sur. Los sitios monumentales del valle de Chincha se encuentran en su apogeo e integran económica y políticamente a gran parte de sus comunidades (Tantaleán et al. 2013; Tantaleán 2016). Lo mismo sucede con los sitios del valle medio y alto de Ica como Ánimas Altas/Ánimas Bajas y Cerrillos (Menzel et al. 1964: 175; Massey 1991; Cook 1999; Van Gijseghem 2006: 426, Bachir Bacha 2017). En esta fase, la representación del «Ser Oculado» se hizo muy importante en el valle de Ica (Menzel et al. 1964: 171, 196; Dwyer 1979: 115; Massey 1991: 343). Las elites ya no son las únicas que visitan los lugares centrales, sino que grupos de personas peregrinan a las huacas importantes de cada zona relevante de la costa sur. Así, se explica la aparente homogeneidad de la cultura material Paracas durante la fase Paracas Tardío.

\section{Desaparición del fenómeno Paracas}

Durante el II siglo a.C. la mayoría de los sitios Paracas comenzaron a ser abandonados o presenciaron la su- 
perposición de estructuras arquitectónicas, intrusión de entierros humanos y/o depósito de materiales diferentes. Entre esos nuevos materiales que aparecieron se destaca la cerámica de un estilo monocromo de gran finura conseguida gracias a innovaciones en su cocción (Tantaleán et al. 2013; Reindel 2009: 450). Este estilo cerámico es conocido en la literatura arqueológica como Topará (Wallace 1986; Paul 1991; Proulx 2008). Además, como en las Necrópolis de Paracas, en otros sitios aparece una tradición textil que, aunque tiene antecedentes en Cavernas, se convierte en un arte altamente desarrollado e iconográficamente complejo.

Según las investigaciones en el valle de Palpa a mediados del siglo 100 a. C. se acentuó el proceso de sequedad de los valles de la vertiente del Pacífico con un retroceso de la vegetación (Isla 2005; Llanos 2017: 182). Esta sequedad pudo haber mermado la capacidad de las elites para gobernar e incluso impedir el desarrollo de las comunidades Paracas, principalmente agrícolas. Por su parte, el análisis del patrón de asentamiento realizado por Cook (1999: 85) en el valle de Ica señala que hacia finales de la época Paracas existiría un escenario de conflicto.

En suma, se puede decir que hacia finales de la fase Paracas Tardío existieron diferentes escenarios medioambientales, económicos y políticos locales que conllevaron a la desintegración a nivel de valles $y$, por consecuencia, a nivel regional del fenómeno Paracas. Muchos sitios Paracas fueron abandonados, pero otros fueron reocupados por grupos sociales que poseían una cultura material diferente.

\section{Comentarios finales}

En el primer milenio antes de nuestra era una serie de comunidades de los valles de la costa sur desarrollaron sus propias historias y materialidades, aunque compartieron una serie de elementos ideológicos y religiosos que los hace reconocibles como parte de la denominada "cultura Paracas». Como este artículo ha mostrado y explicado, el fenómeno Paracas es mucho más complejo y no se puede reducir y simplificar mediante la noción de cultura. Por el contrario, lo que se observa mediante la investigación arqueológica reciente es que existieron una serie de prácticas y relaciones sociales fluidas y cambiantes a lo largo de este milenio mediante los cuales las comunidades se organizaron tanto interna como externamente para poder reproducirse económica, política e ideológicamente.

Así, en cada valle de la costa sur peruana se generó una expresión social manifestada en su forma de asentamiento y arquitectura, aunque compartieron una serie de prácticas y motivos religiosos e ideológicos que los cohesionaron en ciertos lugares centrales. Si bien el lugar central de Cerro Colorado concentró una importante cantidad de enterramientos y un asentamiento humano de la época, cada valle generó sus propias comunidades con sus respectivas expresiones arquitectónicas. De entre estos valles de la costa sur, resaltan Chincha, Ica y Palpa, los cuales concentraron una importante cantidad de asentamientos, arquitectura y materialidad social notable. De hecho, estas concentraciones de arquitectura nos permiten establecer que en cada uno de estos valles se generaron una serie de comunidades con sus respectivas elites y lideres que establecieron sus propias practicas políticas y económicas, pero que también fueron interdependientes con las demás elites y comunidades cercanas. De esta manera, este artículo ha mostrado un panorama mucho más complejo que el que poseíamos y que espera pueda ser enriquecido en los próximos años mediante más trabajos de campo arqueológico en las zonas conocidas y mencionadas aquí y en las nuevas por descubrir e investigar.

\section{Referencias bibliográficas}

Bachir Bacha, Aïcha (2017). El Edificio de los Frisos de Ánimas Altas. Ser Paracas en el Valle Bajo de Ica. Boletin de Arqueología PUCP 22:191-225.

Bachir Bacha, Aïcha (2018). Paracas, Geography and Culture of. En C. Smith (ed.): Encyclopedia of Global Archaeology. Springer, Cham.

Bachir Bacha, Aïcha y Daniel Llanos (2013) ¿Hacia un Urbanismo Paracas en Ánimas Altas/Ánimas Bajas (Valle de Ica)? Boletín de Arqueología PUCP 17:169-204.

Balbuena, Lucía (2013). Evidencias Paracas en los Valles de Pisco y Mala. Boletín de Arqueología PUCP 17:57-75. Beresford-Jones, David, Carmela Alarcón, Susana Arce, Alex Chepstow-Lusty, Oliver Whaley, Fraser Sturt, Manuel Gorriti, Oscar Portocarrero y Lauren Cadwallader (2009). Ocupación y Subsistencia del Horizonte 
Temprano en el Contexto de Cambios Ecológicos de Largo Plazo en las Cuencas de Samaca y Ullujaya, Valle Bajo de Ica. Boletín de Arqueología PUCP 13:237-257. Browman, David (1970). Early Peruvian Peasants: The Culture History of a Central Highlands Valley. Tesis doctoral, Departamento de Antropología, Harvard University, Cambridge.

Burger, Richard (1992). Chavin and the Origins of Andean Civilization. Thames and Hudson, Londres.

Cadwallader, Lauren, Susana Arce, Tamsin O'Connell, Alexander Pullen y David Beresford-Jones (2015). Dating the Dead: New Radiocarbon Dates from the Lower Ica Valley, South Coast Peru. Radiocarbon 57(5):765-773.

Cadwallader, Lauren, Susana Arce Torres, Alexander Pullen y David Beresford-Jones (2018). Algunas Contribuciones a la Comprensión de la Transición Paracas-Nasca, Samaca, Valle Bajo de Ica. Boletín de Arqueología PUCP 25:147-171.

Castro-Martínez, Pedro, Trinidad Escoriza-Mateu, Andrea González-Ramírez, Samy Irazábal, Arturo Saez-Sepúlveda y Víctor Salazar (2016). Proyecto de Investigación Arqueológica La Puntilla: Últimas Aportaciones de las Excavaciones en Extensión en los Asentamientos de El Trigal (Nasca, Ica, Perú). En Actas del I Congreso Nacional de Arqueología:293-301. Ministerio de Cultura del Perú, Lima.

Cook, Anita (1999). Asentamientos Paracas en el Valle Bajo de Ica. Gaceta Arqueológica Andina, 25: 61-90.

DeLeonardis, Lisa (2005). Early Paracas Cultural Contexts: New Evidence from Callango. Andean Past, 7:27-56.

Dulanto, Jalh (2013). Puerto Nuevo: Redes De Intercambio a Larga Distancia Durante la Primera Mitad del Primer Milenio Antes de Nuestra Era. Boletin de Arqueología PUCP 17:103-132.

Dulanto, Jalh y Aldo Accinelli (2013). Disco Verde Cincuenta Años Después de Frédéric Engel: La Primera Temporada de Excavaciones del Proyecto de Investigaciones Arqueológicas Paracas en el Sitio. Boletín de Arqueología PUCP 17:133-150.

Dwyer, Jane (1979). The Chronology and Iconography of Paracas-Style Textiles. En The Junius B. Bird PreColumbian Textile Conference, editado por Ann Pollard Rowe y Anne-Louise Schaffer, pp. 105-128. The Textile Museum and Dumbarton Oaks, Washington, D.C.

Engel, Frederic (1957). Early Sites in the Pisco Valley of Peru: Tambo Colorado. American Antiquity 23(1):34-45.
Engel, Frederic (1966). Paracas. Cien Siglos de Cultura Peruana. Mejía Baca, Buenos Aires.

Engel, Frederic (1991). Un Desierto en Tiempos Prehispánicos. Rio Pisco, Paracas, Rio Ica. Fondation pour l'étude des problèmes de terres arides, Lima.

García, Rubén (2009). Puerto Nuevo y los Orígenes de la Tradición Estilístico-Religiosa Paracas. Boletín de Arqueología PUCP 13: 187-207.

García, Rubén (2010). La Ocupación Paracas en la Bahía de la Independencia de Paracas. En Valle, Luis (ed.): Arqueología y Desarrollo: Experiencias y Posibilidades en el Perú: 53-66. SIAN, Lima.

García, Rubén (2013). Geoglifos Paracas de la Costa Sur: Cerro Lechuza y Cerro Pico. Boletín de Arqueología PUCP 17: 151-168.

García, Rubén y José Pinilla (1995). Aproximación a una Secuencia de Fases con Cerámica Temprana de Paracas. Journal of the Steward Anthropological Society 23(1-2):43-81.

Isla, Johny (2009). From Hunters to Regional Lords: Funerary Practices in Palpa, Peru. En Markus Reindel y Günther Wagner (eds.): New Technologies for Archaeology Multidisciplinary Investigations in Palpa and Nasca, Peru:119-139. Springer, Berlín.

Isla, Johny (2018). Los Geoglifos de la Región de Ica. Estado de la Cuestión y Perspectivas. En More, Gabriel (ed.): Proyecto de Rescate Arqueológico de los Geoglifos de Pampa de Ocas, Paracas, Pisco-Ica: 87-130. Futura, Lima.

Isla, Johny y Markus Reindel (2007). Los Paracas del Sur: Una Nueva Perspectiva desde los Valles de Palpa. En Hilos del Pasado. El Aporte Francés al Legado Paracas: 79-91. Instituto Nacional de Cultura, Lima.

Kaulicke, Peter, Lars Fehren-Schmitz, María KolpGodoy,Patricia Landa, Óscar Loyola, Martha Palma, Elsa Tomasto, Cindy Vergel y Burkhard Vogt (2009). Implicancias de un Área Funeraria del Periodo Formativo Tardío en el Departamento de Ica. Boletín de Arqueología PUCP 13: 89-322.

Kroeber, Alfred (1944). Peruvian Archaeology in 1942. Viking Fund, New York.

Lanning, Edward (1960). Chronological and Cultural Relationships of Early Pottery Styles in Ancient Peru. Tesis doctoral. Departamento de Antropología, University of California, Berkeley.

Massey, Sarah (1986). Sociopolitical Change in the Upper Ica Valley: B.C. 400 to 400 A.D.; Regional States on the 
South Coast of Peru. Tesis doctoral. Departamento de Antropología, University of California, Los Angeles.

Massey, Sarah (1991). Social and Political Leadership in the Lower Ica Valley. En Anne Paul (ed.): Paracas, Art \& Architecture. Object and Context in South Coastal Peru: 315-345. University of Iowa Press, Iowa city.

Matsumoto, Yuichi (2012). Paracas en la Sierra: Interacción Temprana entre la Sierra Centro-Sur y Costa Sur. Peruvian Archaeology 3:33-64.

Mendoza, Edison (2017). Secuencia de Cerámica Paracas en Pallaucha, Vilcashuamán-Ayacucho. Boletín de Arqueología PUCP 22: 91-116.

Orefici, Giuseppe (2012). Cahuachi. Capital Teocrática Nasca. Tomo I. Universidad de San Martín de Porres, Lima.

Paul, Anne (1991). Paracas. An Ancient Cultural Tradition on the South Coast of Peru. En Anne Paul (ed.): Paracas, Art \& Architecture. Object and Context in South Coastal Peru: 1-33. University of Iowa Press, Iowa city.

Peters, Ann (1987/1988). Chongos: Sitio Paracas en el valle de Pisco. Gaceta Arqueológica Andina 16:30-34.

Peters, Ann (2013). Topará en Pisco: Patrón de Asentamiento y Paisaje. Boletín de Arqueología PUCP 17:77-101.

Pezo-Lanfranco, Luis, Delia Aponte y Sabine Eggers (2015). Aproximación a la Dieta de las Sociedades Formativas Tardías del Litoral de Paracas (Costa Sur del Perú): Evidencias Bioarqueológicas e Isotópicas. Nawpa Pacha 35(1):23-55.

Pezzia, Alejandro (1968). Ica y el Perú Precolombino. Tomo I. Arqueología de la Provincia de Ica. Ojeda, Ica.

Proulx, Donald (2008). Paracas and Nasca: Regional Cultures on the South Coast of Peru. En Handbook of South American Archaeology, editado por Helaine Silverman y William H. Isbell:563-585. Springer, New York

Reindel, Markus (2009). Life at the Edge of the DesertArchaeological Reconstruction of the Settlement History in the Valleys of Palpa, Peru. En Markus Reindel y Günther Wagner (eds.): New Technologies for Archaeology Multidisciplinary Investigations in Palpa and Nasca, Peru:439-461. Springer, Berlín.

Reindel, Markus y Johny Isla (2006). Evidencias de Culturas Tempranas en los Valles de Palpa. Boletín de Arqueología PUCP 10:237-283.

Reindel, Markus y Johny Isla (2013). Jauranga: Una Aproximación a la Ocupación Paracas en los Valles de Palpa. Boletín de Arqueología PUCP, 17:231-262.
Reindel, Markus y Johny Isla (2017). Nuevo Patrón Arquitectónico Paracas en Lucanas, Sierra Sur del Perú. Boletín de Arqueología PUCP, 22:227-254.

Reindel, Markus y Johny Isla (2018). De Paracas a Nasca: Nuevas Evidencias Desde la Vertiente Occidental de la Sierra de Lucanas, Ayacucho. Boletín de Arqueología PUCP 25:229-254.

Sakai, Masato, Jorge Olano, Yuichi Matsumoto y Hiraku Takahashi (2014). Centros de Lineas y Cerámica en las Pampas de Nasca, Perú, 2010. Yamagata University Press, Yamagata.

Sakai, Masato, Jorge Olano y Hiraku Takahashi (2019). Centros de Lineas y Cerámica en las Pampas de Nasca, Perú, Hasta el Año 2018. Yamagata University Press, Yamagata.

Sawyer, Alan (1966). Ancient Peruvian Ceramics: The Nathan Cummings Collection. The Metropolitan Museum of Art, New York.

Silverman, Helaine (1991). The Paracas Problem: Archaeological Perspectives. En Paracas, Art and Architecture: Object and Context in South Coastal Peru, editado por A. Paul, pp. 349-415. University of Iowa Press, Iowa City.

Silverman, Helaine (1994). Paracas in Nazca: New Data on the Early Horizon Occupation of the Rio Grande de Nazca Drainage, Peru. Latin American Antiquity, 5(4): 359-382.

Silverman, Helaine (1997). The First Field Season of Excavations at the Alto del Molino Site, Pisco Valley, Peru. Journal of Field Archaeology 24(4):441-457.

Stanish, Charles, Henry Tantaleán, Benjamin Nigra y Laura Griffin (2014). A 2,300-Year-Old Architectural and Astronomical Complex in the Chincha Valley, Peru. PNAS 111(20): 7218-7223.

Splitstoser, Jeffrey, Dwight Wallace y Mercedes Delgado (2009). Nuevas Evidencias de Textiles y Cerámica de la Época Paracas Temprano en Cerrillos, Valle de Ica, Perú. Boletín de Arqueología PUCP 13:209-235.

Tabío, Ernesto (1965). Excavaciones en la Costa Central del Perú (1955-58). Academia de Ciencias, Departamento de Investigaciones Antropológicas, Instituto Nacional de Cultura, La Habana.

Tantaleán, Henry (2016). Paisajes Rituales y Políticos Paracas en el Valle de Chincha, Costa Sur del Perú. Latin American Antiquity 27(4):479-496.

Tantaleán, Henry, Charles Stanish, Michiel Zegarra, Kelita Pérez y Ben Nigra (2013). Paracas en el Valle 
de Chincha: Nuevos Datos y Explicaciones. Boletín de Arqueología PUCP 17:31-56.

Tantaleán, Henry, Alexis Rodríguez, Irving Aragonéz, Boris Orccosupa y José Román (2019) Pozuelo: The Earliest Ceramic in Chincha Valley. Ponencia presentada en la 84th Annual Meeting of the Society for American Archaeology, abril 10-14, Albuquerque.

Tantaleán, Henry y Charles Stanish (eds.) (2017). Cerro del Gentil: Un Sitio Paracas en el valle de Chincha, Costa Sur del Perú. PACH press, Lima.

Tello, Julio C. (2009). Paracas Cavernas. Cuaderno de Investigación del Archivo Tello 7. C. Sotelo Sarmiento (ed.). Museo de Arqueología y Antropología de la Universidad Nacional Mayor de San Marcos, Lima.

Tello, Julio C. y Toribio Mejía Xesspe (2005[1959]). Paracas Primera Parte. Universidad Nacional Mayor de San Marcos, Lima.

Tello, Julio C. y Toribio Mejía Xesspe (1979). Paracas Segunda Parte: Cavernas y Necrópolis. Universidad Nacional Mayor de San Marcos, Lima.

Uhle, Max (1914). The Nazca Pottery of Ancient Peru. Proceedings of the Davenport Academy of Sciences 13:1-16.

Unkel, Ingmar, Markus Reindel, Hermann Gorbahn, Johny Isla, Bernd Kromer y Volker Sossna (2012). A Comprehensive Numerical Chronology for the Pre-Columbian Cultures of the Palpa Valleys, South Coast of Peru. Journal of Archaeological Science 39(7):2294-2303.

Valdez, Lidio (2012). Las Ocupaciones Tempranas del Valle de Acarí, Perú. Ponencia presentada en el Simposio
«Paracas-Nasca: Una Época «Transicional» del Formativo Tardío, Costa Sur de los Andes Centrales.». Disponible en: https://www.academia.edu/31595582/ LAS_OCUPACIONES_TEMPRANAS_DEL_ VALLE_DE_ACAR\%C3\%8D_PER\%C3\%9A

Van Gijseghem, Hendrik (2006). A Frontier Perspective on Paracas Society and Nasca Ethnogenesis. Latin American Antiquity 17(4):419-444.

Wallace, Dwight (1962). Cerrillos, an Early Paracas Site in Ica, Perú. American Antiquity 27(3):303-314.

Wallace, Dwight (1971). Sitios Arqueológicos del Perú (Segunda Entrega): Valles de Chincha y de Pisco. Arqueológicas, 13:3-131.

Wallace, Dwight (1986). The Topará Tradition: An Overview. En Sandweiss, Daniel y Peter Kvietok (eds.): Perspectives on Andean Prehistory and Protohistory:35-47. Latin American Studies Program. Cornell University, Cornell.

Wallace, Dwight (1991). A Technical and Iconographic Analysis of Carhua Painted Textiles. En Anne Paul (ed.): Paracas Art and Architecture: Object and Context in South Coastal Peru: 61-109. University of Iowa Press, Iowa City.

Würster, Wolfgang (1984). Asentamientos Prehispánicos en el Valle de Topará. Historia y Cultura 17: 7-16.

Young, Michelle (2017). De la Montańa al Mar: Intercambio Entre la Sierra Centro-Sur y la Costa Sur Durante el Horizonte Temprano. Boletin de Arqueología PUCP 22:9-34. 